\title{
SINGULAR VIETORIS-BEGLE THEOREMS FOR RELATIONS
}

\author{
BY
}

\author{
D. G. BOURGIN AND ROBERT M. NEHS
}

\begin{abstract}
The Vietoris-Begle theorem with singularities, for three spaces $X, Y, T$, is extended to the case that a closed relation replaces a continuous map and more generally to set valued maps. The developments are carried out based on modification of the topology of $T$ so that in general it is no longer even Hausdorff. This entails interpretation of dimension of singulars sets in terms of considertions in $Y$ rather than $T$. The techniques are those of sheaf and spectral sequence theory.
\end{abstract}

Introduction. The Vietoris-Begle theorem has been generalized by different methods and under somewhat different hyppotheses to possibly finite ranges of grades not necessarily including 0 in [1], using collections of cone caps over singularities, and in [2] using spectral sequences, for the case that all maps are single valued. The developments in this paper are concerned with Vietoris-Begle type theorems associated with a relation $R \subset Y \times T$. For instance, $R$ may be the graph of a set valued transformation. We shall invariably suppose $Y$ is at least paracompact. We remark that in much of this work $T$ may be viewed as a parameter space.

The cone caps method of [1] seems to require that the relative covering dimension of singularities be 0 . We have therefore chosen a spectral sequence technique. In particular, an analogue of the Leray sheaf is required. How to define this sheaf is not obvious. As a preliminary, as well as providing motivation for the procedure introduced, we consider the association of a sheaf on one space with an assigned sheaf on another. The difficulties are, perhaps, better recognized by presenting some possible definitions of such associated sheaves.

We shall have occasion to refer to the two well-known conditions for a presheaf to be a sheaf-labelled $S_{1}$ and $S_{2}$ in [7, p. 5 or 3].

A direct assault immediately encounters problems. Thus the presheaf over $T$ defined by (cf. (3)) $U \rightarrow \Gamma(G(U), \mathscr{A})$ for $U$ open in $T$ and $\mathscr{A}$ a sheaf over $Y$ has the defect that in general $S_{2}$ is not satisfied. For instance, suppose

$$
G\left(t_{1}\right) \cap G\left(t_{2}\right) \neq \varnothing, \quad t_{1} \neq t_{2} .
$$

Write $V=V_{1} \cup V_{2}$ where $V_{1}$ and $V_{2}$ are disjunct open neighborhoods of $t_{1}$ and $t_{2}$. Let

$$
\gamma_{1} \in \Gamma\left(G\left(V_{1}\right), \mathscr{A}\right), \quad \gamma_{2} \in \Gamma\left(G\left(V_{2}\right), \mathscr{A}\right)
$$

Received by the editors December 14, 1982 and, in revised form, August 24, 1983.

1980 Mathematics Subject Classification. Primary 55N30, 55T25; Secondary 54A10, 54C60.

Key words and phrases. Vietoris-Begle theorem, sheaf, spectral sequence, paracompact, graph, identification topology. 
No compatibility condition enters, since $V_{1} \cap V_{2}=\varnothing$. Accordingly, if $G\left(V_{1}\right) \cap$ $G\left(V_{2}\right) \neq \varnothing$, there need be no corresponding $\gamma \in \Gamma(G(V), \mathscr{A})$ for which $\gamma \mid G\left(V_{i}\right)=$ $\gamma_{i}, i=1,2$. (Moreover, even if some normalizing condition could piece together the $\gamma_{i}$ 's in special cases, there would remain the problem of continuity of the $\gamma$ 's since $G$ is not necessarily open.)

This obstacle can be skirted by introducing the graph of the set-valued transformation $G$ from $T$ to $Y$ with $p$ and $q$ projection maps. Thus

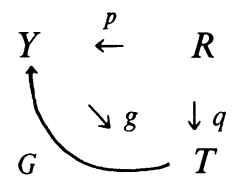

where

$$
p q^{-1} t=G(t), \quad q p^{-1} y=g(y)
$$

so, in a set transformation sense, $g=G^{-1}$.

With $\mathscr{A}$ a sheaf over $Y$ and $\mathscr{B}$ a sheaf over $T$, there result the associated sheaves $g_{R}(\mathscr{A})$ over $T$ and $G_{R}(\mathscr{B})$ over $Y$ according to the following scheme:

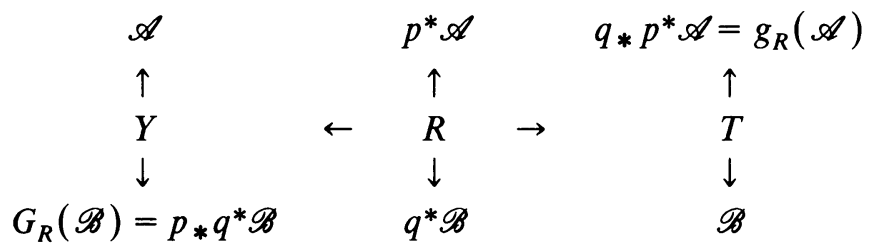

Though $g_{R}(\mathscr{A})$ and $G_{R}(\mathscr{B})$ are sheaves, the section $s \in g_{R}(U)$, for instance, involves $R$ and not $Y$ alone. The presheaf is defined by

$$
U \rightarrow g_{R}(U)=\left\{s: q^{-1}(U) \rightarrow \mathscr{A} \mid s(y, t) \in \mathscr{A}_{y}\right\}
$$

for $U$ open in $T$. Then the presheaf $U \rightarrow \Gamma(G(U), \mathscr{A}(U))$ maps monomorphically into $g_{R}(U)$.

Let $T_{y}=\{t \mid(y, t) \in R\} \cap U$. Then $s(y, t)$ ought be the same member of $\mathscr{A}_{y}$ for all $t \in T_{y}$ which almost demands the restriction that for $y \in G(U), T_{y}$ is connected. Then with $p$ restricted to $q^{-1}(U), s p^{-1}$ may not be continuous.

Another difficulty with both $g_{R}(\mathscr{A})$ and $G_{R}(\mathscr{B})$ is the presence of the inverse sheaves $p^{*} \mathscr{A}$ and $q^{*} \mathscr{B}$ since a flabby resolution of $\mathscr{A}(\mathscr{B})$ may not yield a flabby resolution of $g_{R}(\mathscr{A})\left(G_{R}(\mathscr{B})\right)$. To blunt this objection, one can start with a sheaf $\mathscr{C}$ over $R$ and associate $p_{*} \mathscr{C}$ and $q_{*} \mathscr{C}$. This is not altogether satisfactory either, since usually no natural choice for $\mathscr{C}$ is indicated except, for instance, when $\mathscr{C}$ is a constant sheaf. This special case with stalks an Abelian group so $p_{*} \mathscr{C}$ and $q_{*} \mathscr{C}$ are again constant sheaves with stalks the same groups is the one considered in [1, Theorem 9.1] under the added condition that $Y$ and $T$ are identified. An alternative is to introduce two independent sheaves, $\mathscr{A}$ over $Y$ and $\mathscr{B}$ over $T$. Then $\mathscr{A} \oplus \mathscr{B}$ is a sheaf over $Y \times T$ and $\mathscr{C}$ can be taken as

$$
\mathscr{C}=(\mathscr{A} \oplus \mathscr{B}) \mid R .
$$

However, the introduction of two independent sheaves seems artificial. 
In this work we treat the consequences of dealing with a single assigned sheaf over $Y$. Our approach necessitates introducing some radical measures, notably retopologizing $T$ (if it already bears a topology). Generally, in this new topology $T$ is no longer Hausdorff, though this is not an anomaly in sheaf theory. Furthermore, certain restrictions on subsets of $Y$ connected with our definition of saturation seem called for. These measures are not without cost. Specifically, in assessing the influence of singular sets, an index, referred to as dimension, enters. The analogous index in [1 and 2] is in terms of a relative covering dimension, and so may be viewed as constructively, though perhaps not always practically, defined. However, because of the modified topology of $T$, the dimension definition may be difficult to apply, and we are constrained to seek expression of our conclusions in terms of $Y$ dimension. A number of examples are included to bring out the reasonable, or sometimes compelling, nature of our restrictions. Among other minor novelties may be cited, for instance, the introduction of determining and $\mathbf{z}$-sets, or our treatment of the spectral sequences argument. The latter demonstration is of the type in [ 2 and 3 ], but is notably more direct and perspicuous by reason of inferences from diagram 5.5.1.

One application is to a generalization covering several aspects of a problem considered in $[4,5$ and 6] where $Y=T$. In part, such a generalization using the collection of cones technique is given in [1] for compact spaces and constant sheaves.

An overview of the general plan of exposition consisting of excerpts and paraphrasing of the substance of some results follows. We consider

$$
X \stackrel{f}{\rightarrow} Y \stackrel{g}{\rightarrow} T
$$

where $f$ is a closed, continuous surjection of paracompact spaces and $g$ is set valued.

$\$ 1$ develops the functorial and spectral sequence apparatus, basic for the rest of the paper. In particular, the functors $\mathbf{g}$ and $\mathbf{g f}$ are defined acting on the sheaf categories $\mathfrak{S}(Y)$ and $\mathfrak{S}(X)$ with the aim of achieving Leray type sheaves and spectral sequences. Thus, for instance,

PROPOSITION 1.3. There is a spectral sequence with

$$
E_{2}^{p q}=H^{p}\left(T, \mathscr{H}^{q}(\mathbf{g}, \mathscr{A})\right)
$$

and target $H^{*}(Y, \mathscr{A})$.

The underlying sheaf cohomorphisms are

$$
\psi: \mathscr{A} \rightarrow f^{*} \mathscr{A}
$$

and

$$
\psi^{*}: H^{*}(Y, \mathscr{A}) \rightarrow H^{*}\left(X, f^{*} \mathscr{A}\right) .
$$

A fundamental conclusion is

THEOREM 1.7. There are spectral sequences $E_{r}^{p q}$ and $\hat{E}_{r}^{p q}$ with targets $H^{*}(Y, \mathscr{A})$ and $H^{*}\left(Y, f^{*} \mathscr{A}\right)$ where
$(1.7)(\mathrm{a})$
$E_{2}^{p q}=H^{p}\left(T, \mathscr{H}^{q}(\mathbf{g}, \mathscr{A})\right)$,
$(1.7)(b)$
$\hat{E}_{2}^{p q}=H^{p}\left(T, \mathscr{H}^{q}\left(\mathbf{g f}, f^{*} \mathscr{A}\right)\right)$. 
There is a homomorphism $\lambda_{r}^{p q}$ on $E_{r}^{p q}$ so that (cf. (1.53))

$$
\begin{aligned}
& (1.7)(\mathrm{c}) \quad\left\{\lambda_{2}^{p q}, \psi^{*}\right\}:\left\{E_{r}^{p q}, H^{*}(Y, \mathscr{A})\right\} \rightarrow\left\{\hat{E}_{r}^{p q}, H^{*}\left(X, f^{*} \mathscr{A}\right)\right\}, \\
& (1.7)(\mathrm{d}) \quad \lambda_{2}^{p q}=H\left(\mathbf{g}(\psi)^{*}\right): H^{p}\left(T, \mathscr{H}^{q}(\mathbf{g}, \mathscr{A})\right) \rightarrow H^{p}\left(T, \mathscr{H}^{q}\left(\mathbf{g f}, f^{*} \mathscr{A}\right)\right) .
\end{aligned}
$$

In $\S 2$, if $G$ is the inverse of $g$ and if $g^{-1}(t)$ is denoted by $G_{t}$, then the collection of nonempty subsets of $Y,\left\{G_{t} \mid t \in T\right\}$ plays a central role. Moreover, the order of definition can be usefully reversed so that starting with such a collection of subsets, a set-valued $g$ can be defined. A subset $B \subset Y$ is saturated if it is composed of $G_{t}$ sets. Under suitable restrictions detailed in Definition $2.1,\left\{G_{t} \mid T\right\}$ is a determining collection. The totality of all closed sets $B$ in $Y$ with saturated complements constitutes $\Re$. Evidently

LEMMA 2.4. $\Re$ and $g(\Re)$ are closed under finite unions.

We impose a possibly new, generally non-Hausdorff topology $\mathfrak{I}_{g}$ on $T$ by choosing as the basis of open sets those of the form $B_{g}=\{U \mid \tilde{U} \in g(\Re)\}$. A specially significant consequence of this topology in applications is

2.5(b) If $V_{1}$ and $V_{2}$ are open subsets of $T$, then $G\left(V_{1} \cap V_{2}\right)=G\left(V_{1}\right) \cap G\left(V_{2}\right)$.

$\$ 3$ contains examples emphasizing the natural qualifications for determining sets and various concomitants of the topology $\mathfrak{I}_{g}$.

In $\$ 4$ the abstractly defined functors $\mathrm{g}$ and gf of $\$ 1$ are given concrete realizations by use of $\mathfrak{T}_{g}$. On restricting the homomorphisms of (1.5.1) and (1.5.2) to $G_{t}$ and on writing (cf. 4.6) $\psi_{G_{t}}^{*}: H^{*}\left(G_{t}, \mathscr{A}\right) \rightarrow H^{*}\left(f^{-1}\left(G_{t}\right), f^{*} \mathscr{A}\right)$,

PROPOSITION 4.7. For any $t, \psi_{G_{t}}^{*}$ is a monomorphism, epimorphism or isomorphism if and only if these assertions are true for $g(\psi)^{*}(t)$.

In $\S 5$ we let $\psi^{*}: E^{*} \rightarrow \hat{E}^{*}$ be a homomorphism of graded groups. Write $(n, N)$ if the homomorphism is an isomorphism for $n<p<N$ and write $[n, N)$ if $\psi^{*}$ is also surjective for $p=n,(n, N]$ if $\psi^{*}$ is injective for $p=N$ and $[n, N]$ if both these conditions maintain.

Two key theorems here are essential:

THEOREM 5.5. If $E_{r}^{p q}=\hat{E}_{r}^{p q}=0$ for $p<0$ or $q<0$, and if:

5.5(a) $\lambda_{2}^{p q}$ is an isomorphism for $n<p+q<N$,

5.5(b) $\lambda_{2}^{p q}$ is surjective for $p+q=n$, and

5.5(c) $\lambda_{2}^{p q}$ is injective for $p+q=N$ and $q \leqslant N-2$, then $\psi^{*}$ is an $[n, N)$ homomorphism.

If $\lambda_{2}^{p q}$ is injective for $p+q=N$, then $\psi^{*}$ is an $[n, N]$ homomorphism.

THEOREM 5.6. If:

5.6(a) $\lambda_{2}^{p q}$ is surjective for $n \leqslant p+q<N+p, 0<p$,

5.6(b) $\lambda_{2}^{p q}$ is injective for $n \leqslant p+q<N+p, 1<p$, and

5.6(c) $\psi^{*}$ is an $[n, N]$ homomorphism, then

5.6(d) $\lambda_{2}^{0 q}$ is an $[n, N]$ homomorphism,

5.6(e) $\lambda_{2}^{1 q}$ is an $[n-1, N)$ homomorphism. 
$\$ 6$ introduces a cohomological relative dimension function $d_{q}=\operatorname{dim}_{T} M_{q}$ for the singular set $M_{q}$ defined by

$$
M_{q}=\left\{t \mid \psi_{G_{t}}^{*}: H^{q}\left(G_{t}, \mathscr{A}\right) \rightarrow H^{q}\left(f^{-1}\left(G_{t}\right), f^{*} \mathscr{A}\right) \text { is not an isomorphism }\right\} .
$$

A subset $B \subset Y$ is q-nonsingular if $\psi_{B}^{q^{*}}$ is an isomorphism in

$$
\psi_{B}^{q^{*}}: H^{q}(B, \mathscr{A}) \rightarrow H^{q}\left(f^{-1} B, f^{*} \mathscr{A}\right) .
$$

If $B$ is $q$-nonsingular for $n<q<N, B$ is ( $n, N)$ nonsingular. If besides $\psi^{n^{*}}$ is onto, then $B$ is $[n, N)$ nonsingular, if $\psi^{N^{*}}$ is a monomorphism, $B$ is $(n, N]$ nonsingular.

A generalized Vietoris-Begle theorem is

THEOREM 6.3. If

$$
\text { (6.3)(a) } \quad 1+d_{q}+q \leqslant n, \quad 0 \leqslant q<N,
$$

then $\psi^{*}: H^{*}(Y, \mathscr{A}) \rightarrow H^{*}\left(X, f^{*} \mathscr{A}\right)$ is an $[n, N)$ homomorphism.

Furthermore, if

$$
\psi_{G_{t}}^{*}: H^{N}\left(G_{t}, \mathscr{A}\right) \rightarrow H^{N}\left(f^{-1}\left(G_{t}\right), f^{*} \mathscr{A}\right)
$$

is injective for every $t$, then $\psi^{*}$ is an $[n, N]$ homomorphism.

In the standard Vietoris-Begle theorem, the points of $Y$ are nonsingular while in our generalizations the sets $g^{-1}(t)$ are nonsingular. $\$ 6$ includes examples in which the nonsingular sets $g^{-1}(t)$ are not free of point singularities and also cases showing the necessity of our added conditions or those of [1] as compared with those in the simpler situation treated in [5 and 6]. To treat an inverse Vietoris-Begle type theorem we introduce the concept of a $\mathrm{z}$-set for $M \subset T$, naturally characterized by the stipulation that $H^{0}(T, \mathscr{A}) \neq 0$ for nontrivial sheaves on $T$ concentrated on $M$. It is not necessarily true that 0 -dimensional sets are $\mathbf{z}$-sets. We show

THEOREM 6.9. Suppose that

$$
\begin{gathered}
d_{q}+q+1 \leqslant n \text { for } 0 \leqslant q \leqslant n-1, \\
M_{q} \text { is a } \mathbf{z}-\text { set and } d_{q} \leqslant 0 \text { for } n \leqslant q<N, \\
\psi^{*}: H^{q}(Y, \mathscr{A}) \rightarrow H^{q}\left(X, f^{*} \mathscr{A}\right) \text { is an }[n, N] \text { homomorphism. }
\end{gathered}
$$

Then $\psi_{G_{t}}^{*}$ is an $[n, N)$ homomorphism for all $t$. Furthermore, if $\left\{t \mid \psi_{G_{t}}^{*}\right.$ is not injective $\}$ is a z-set, it is vacuous, so $\psi_{G_{t}}^{*}$ is an $[n, N]$ homomorphism.

$\$ 7$ has as its principal objective the replacement of the cohomological dimensional restrictions on the singular set in $T$, which may be difficult to verify, by standard, covering space dimensional restrictions on subsets of the paracompact space $Y$. A preliminary result is

Proposition 7.1. If $B \subset T$ consists of a finite number of closed points, then $B$ is a zero-dimensional $\mathbf{z}$-set.

For more general situations it is desireable to introduce a canonical determining collection $\left\{G_{t}^{1} \mid t^{1} \in T^{1}\right\}$. Thus for $B$ closed in $Y$ suppose $U$ open $\supset B$. Let $T^{1}=T_{0}+(U-B)+\{a\}$ where $T_{0}=\left\{t \mid G_{t} \cap \tilde{U} \neq \varnothing\right\}$ and $\{a\}$ is a special ideal point. It is then the case that 
Proposition 7.3. The determining set $\left\{G_{t} \mid t \in T\right\}$ can be replaced by $\left\{G_{t}^{1} \mid t^{1} \in T^{1}\right\}$ where

$$
\begin{gathered}
G_{a}^{1}=B, \\
G_{y}^{1}=\{y\} \quad \text { for } y \in U-B, \\
G_{t_{0}}^{1}=G_{t_{0}}-U \text { for } t_{0} \in T_{0} .
\end{gathered}
$$

Furthermore, if the points of $U-B$ are $(-1, N)$ nonsingular, then for $t_{0} \in T_{0}, G_{t_{0}}^{1}$ is $[n, N]$ nonsingular whenever $G_{t_{0}}$ is.

Finally, we can state

THEOREM 7.6. Suppose each $G_{t}$ is $[n, N]$ nonsingular and $G_{t}$ is $(-1, n]$ nonsingular for the subset $T_{1}$. Let $B_{q}$ be the set of q-singular points in $\bigcup_{T-T_{1}} G_{t}$ and let $B=\bigcup_{q=0}^{N-1} B_{q}$. Let $d_{q}=r d_{Y} B_{q}$, the relative dimension of $B_{q}$ in $Y$ [8]. If $\bar{B} \subset U$, where $U$ is open and all $(-1, N)$ singular points of $U$ are in $B$, and if

$$
\max _{0 \leqslant q<N}\left(2+d_{q}+q\right) \leqslant n
$$

then $\psi^{*}: H^{q}(Y, \mathscr{A}) \rightarrow H^{q}\left(X, f^{*} \mathscr{A}\right)$ is an $[n, N]$ homomorphism.

1. Functorial formulation. Sheaves are understood to be sheaves of Abelian groups. The sheaf category over the space $W$ is denoted $\subseteq(W)$. The letters $X, Y$ invariably designate topological spaces generally taken paracompact $T$ generally is a topological space also, but in special instances is merely a set.

Let $\mathrm{g}: \mathfrak{S}(Y) \rightarrow \mathfrak{S}(T)$ be a functor subject to:

$1.1(\mathrm{a}) \mathrm{g}$ is a left exact, additive covariant functor,

$1.1(\mathrm{~b}) \mathbf{g}(\mathscr{A})$ is flabby whenever $\mathscr{A}$ is flabby,

1.1(c) there is a natural equivalence of functors $\Gamma(Y, \cdot) \stackrel{\approx}{\rightarrow} \Gamma(T, \mathbf{g}(\cdot))$.

Let $f: X \rightarrow Y$ be a continuous surjection, and let gf: $\mathfrak{S}(X) \rightarrow \mathfrak{S}(T)$ denote a functor also subject to 1.1(a)-(c). The functor pair $\mathbf{g}$, gf is $f$-compatible when:

1.1(d) If $\lambda$ is a sheaf $f$-cohomomorphism

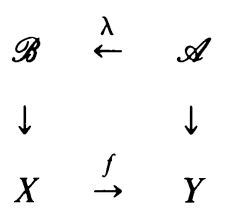

there is a sheaf homomorphism $\mathbf{g}(\lambda): \mathbf{g}(\mathscr{A}) \rightarrow \mathbf{g f}(\mathscr{B})$.

1.1(e) If the diagram

$$
\begin{array}{lll}
\mathscr{A} & \stackrel{\lambda}{\rightarrow} & \mathscr{B} \\
\downarrow \alpha & & \downarrow \beta \\
\mathscr{A}_{1} & \stackrel{\lambda_{1}}{\rightarrow} & \mathscr{B}_{1}
\end{array}
$$


is commutative, where $\mathscr{A}, \mathscr{A}_{1}$ are sheaves over $Y ; \mathscr{B}, \mathscr{B}_{1}$ are sheaves over $X ; \alpha, \beta$ are sheaf homomorphisms and $\lambda, \lambda_{1}$ are $f$-cohomomorphisms, then the induced diagram

$$
\begin{array}{ccc}
\mathbf{g}(\mathscr{A}) & \stackrel{\mathrm{g}(\lambda)}{\rightarrow} & \mathbf{g f}(\mathscr{B}) \\
\downarrow \mathbf{g}(\alpha) & & \downarrow \mathbf{g f}(\beta) \\
\mathbf{g}\left(\mathscr{A}_{1}\right) & \stackrel{\mathrm{g}\left(\lambda_{1}\right)}{\rightarrow} & \mathbf{g f}\left(\mathscr{B}_{1}\right)
\end{array}
$$

commutes.

1.1(f) For the $f$-cohomorphism in (1.1.1), there is a commutative diagram

$$
\begin{array}{cccc}
\Gamma(Y, \mathscr{A}) & \stackrel{\approx}{ } & \Gamma(T, \mathbf{g}(\mathscr{A})) \\
\downarrow \Gamma(\lambda) & & \downarrow \mathbf{g}(\lambda) \\
\Gamma(X, \mathscr{B}) & \stackrel{\approx}{\rightarrow} & \Gamma(T, \mathbf{g f}(\mathscr{B}))
\end{array}
$$

with the isomorphisms guaranteed by $1.1(\mathrm{c})$.

The restrictions in 1.1 are satisfied, of course, when $\mathbf{g}(\mathscr{A})$ is the direct image of $\mathscr{A}$ induced by a continuous surjection $g: Y \rightarrow T$. However, our interest is in a correspondence $g$ which is not constrained to be either single valued or continuous, but which, together with the composition $g f$, yields functors $\mathbf{g}$ and gf characterized above.

The functor $\mathbf{g}$ applied to

$$
0 \rightarrow \mathscr{A} \rightarrow \mathscr{C}^{0} \rightarrow \mathscr{C}^{1} \rightarrow \cdots,
$$

a flabby resolution of the sheaf $\mathscr{A}$ over $Y$, yields a cochain complex

$$
\mathbf{g}\left(\mathscr{C}^{0}\right) \rightarrow \mathbf{g}\left(\mathscr{C}^{1}\right) \rightarrow \mathbf{g}\left(\mathscr{C}^{2}\right) \rightarrow \cdots .
$$

Then

$$
\mathscr{H}^{p}(\mathbf{g}, \mathscr{A})=H^{p}\left(\mathbf{g}\left(\mathscr{C}^{*}\right)\right)
$$

is a sheaf over $T$, namely the derived functor of $\mathrm{g}$. The following assertions follow by standard homological algebra techniques.

(1.2.4) $\mathscr{H}^{p}(\mathrm{~g}, \cdot)$ is a covariant functor from $\subseteq(Y)$ to $\subseteq(T)$ determined by $\mathbf{g}$ and is independent of choice of the flabby resolution of $\mathscr{A}$.

$$
\mathscr{H}^{0}(\mathbf{g}, \mathscr{A})=\mathbf{g}(\mathscr{A}) \text {. }
$$

(1.2.6) A short exact sequence $0 \rightarrow \mathscr{A}_{1} \rightarrow \mathscr{A} \rightarrow \mathscr{A}_{2} \rightarrow 0$ determines a long exact sequence

$$
\cdots \rightarrow \mathscr{H}^{p}\left(\mathbf{g}, \mathscr{A}_{1}\right) \rightarrow \mathscr{H}^{p}(\mathbf{g}, \mathscr{A}) \rightarrow \mathscr{H}^{p}\left(\mathbf{g}, \mathscr{A}_{2}\right) \rightarrow \mathscr{H}^{p+1}\left(\mathbf{g}, \mathscr{A}_{1}\right) \rightarrow \cdots .
$$

Proposition 1.3. There is a spectral sequence with

$$
E_{2}^{p q}=H^{p}\left(T, \mathscr{H}^{q}(\mathbf{g}, \mathscr{A})\right)
$$

and target $H^{*}(Y, \mathscr{A})$. 
In the commutative diagram

$$
\begin{aligned}
& \mathbf{g}(\mathscr{A}) \\
& 0 \rightarrow \mathbf{g}\left(\mathscr{C}^{0}\right) \quad \rightarrow \quad \mathscr{B}^{00} \stackrel{2^{d}}{\rightarrow} \quad \mathscr{B}^{01} \quad \rightarrow \cdots \\
& \downarrow \quad \downarrow_{1} d, \quad \downarrow_{1} d \\
& 0 \rightarrow \mathbf{g}\left(\mathscr{C}^{1}\right) \quad \rightarrow \quad \mathscr{B}^{10} \quad \stackrel{2^{d}}{\rightarrow} \quad \mathscr{B}^{11} \quad \rightarrow \cdots \\
& \begin{array}{ccc}
\downarrow & \downarrow & \downarrow \\
\vdots & \vdots & \vdots
\end{array}
\end{aligned}
$$

the rows are injective resolutions of $\mathbf{g}\left(\mathscr{C}^{p}\right)$ and ${ }_{1} d_{2} d+{ }_{2} d_{1} d=0$. Application of the section functor yields a bigraded complex:

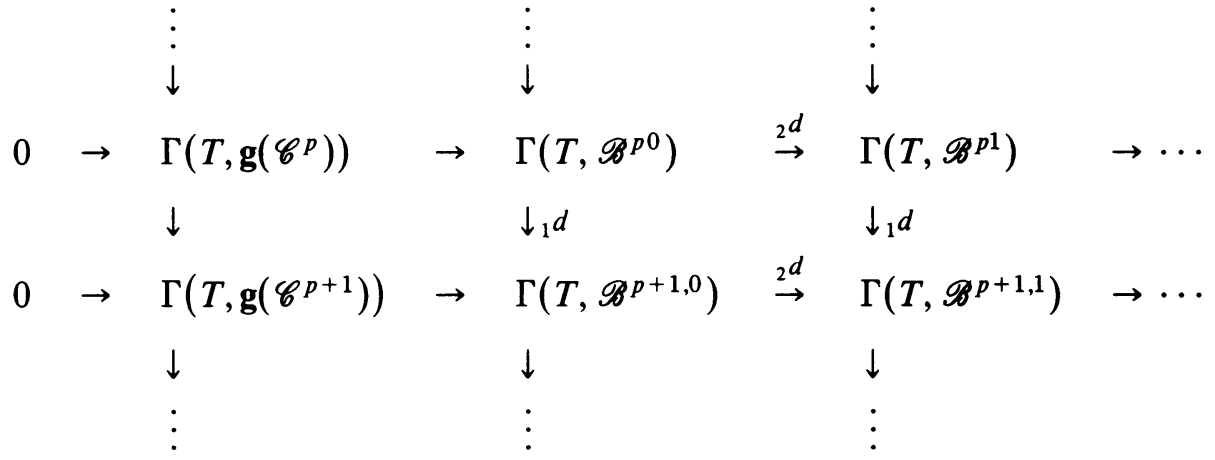

Here $\mathbf{g}\left(\mathscr{C}^{p}\right)$ is flabby $(1.1(\mathrm{~b}))$ and so

$$
{ }_{2} H^{q}\left(\Gamma\left(T, \mathscr{B}^{p_{*}}\right)\right)= \begin{cases}\Gamma\left(T, \mathbf{g}\left(\mathscr{C}^{p}\right)\right) & \text { if } q=0, \\ 0 & \text { if } q>0 .\end{cases}
$$

For the first spectral sequence associated with (1.3.2),

$$
{ }_{1} E_{2}^{p q}={ }_{1} H^{p}\left({ }_{2} H^{q}\left(\Gamma, \mathscr{B}^{* *}\right)\right)= \begin{cases}H^{p}\left(\Gamma\left(T, \mathbf{g}\left(\mathscr{C}^{*}\right)\right)\right) & \text { if } q=0 \\ 0 & \text { if } q>0\end{cases}
$$

Therefore

$$
{ }_{1} E_{\infty}^{p 0}=H^{p}\left(\Gamma\left(T, \mathbf{g}\left(\mathscr{C}^{*}\right)\right)\right),{ }_{1} E_{\infty}^{p q}=0 \text { for } q \neq 0 .
$$

The natural equivalence referred to in 1.1(c) when interpreted for (1.2.1) becomes a commutative diagram:

$$
\begin{array}{cccccc}
\Gamma(Y, \mathscr{A}) & \rightarrow & \Gamma\left(Y, \mathscr{C}^{0}\right) & \rightarrow & \Gamma\left(Y, \mathscr{C}^{1}\right) & \rightarrow \cdots \\
\downarrow \approx & & \downarrow \approx & & & \downarrow \approx \\
\Gamma(T, \mathbf{g}(\mathscr{A})) & \rightarrow & \Gamma\left(T, \mathbf{g}\left(\mathscr{C}^{0}\right)\right) & \rightarrow & \Gamma\left(T, \mathbf{g}\left(\mathscr{C}^{1}\right)\right) & \rightarrow \cdots
\end{array}
$$

Therefore, ${ }_{1} E_{\infty}^{p 0} \approx H^{p}(Y, \mathscr{A})$ which is the target (cf. (1.3.5)). 
For the second spectral sequence associated with (1.3.2)

$$
{ }_{2} E_{2}^{p q}={ }_{2} H^{p}\left({ }_{1} H^{q}\left(\Gamma\left(T, \mathscr{B}^{* *}\right)\right)\right) .
$$

The resolutions in (1.3.1) can be chosen so that

$$
0 \rightarrow{ }_{1} H^{p}\left(\mathbf{g}\left(\mathscr{C}^{*}\right)\right) \rightarrow{ }_{1} H^{q}\left(\mathscr{B}^{* 0}\right) \rightarrow{ }_{1} H^{q}\left(\mathscr{B}^{* 1}\right) \rightarrow \cdots
$$

is an injective resolution of $H^{q}\left(\mathbf{g}\left(\mathscr{C}^{*}\right)\right)=\mathscr{H}^{q}(\mathbf{g}, \mathscr{A})$. Since the section functor is exact on sequences of injective sheaves,

$$
{ }_{1} H^{q}\left(\Gamma\left(T, \mathscr{B}^{* *}\right)\right) \approx \Gamma\left(T,{ }_{1} H^{q}\left(\mathscr{B}^{* *}\right)\right) .
$$

Accordingly

$$
{ }_{2} E_{2}^{p q} \approx{ }_{2} H^{p}\left(\Gamma\left(T,{ }_{1} H^{q}\left(\mathscr{B}^{* *}\right)\right)\right)=H^{p}\left(T, \mathscr{H}^{q}(\mathbf{g}, \mathscr{A})\right) .
$$

The assertions in Proposition 1.3 maintain also for the functor gf and the inverse sheaf $f^{*} \mathscr{A}$. This is to say there is a second spectral sequence with

$$
\hat{E}_{2}^{p q}=H^{p}\left(T, \mathscr{H}^{q}\left(\mathbf{g f}, f^{*} \mathscr{A}\right)\right)
$$

and target $H^{*}\left(X, f^{*} \mathscr{A}\right)$.

The $f$-compatibility conditions $1.1(\mathrm{~d})-(\mathrm{f})$ provide a spectral sequence homomorphism. The natural $f$-cohomomorphism

$$
\psi: \mathscr{A} \rightarrow f^{*} \mathscr{A}
$$

induces a homomorphism

$$
\psi^{*}: H^{*}(Y, \mathscr{A}) \rightarrow H^{*}\left(X, f^{*} \mathscr{A}\right) .
$$

Introduce the canonical flabby resolutions of $\mathscr{A}$ and $f^{*} \mathscr{A}$ and the corresponding lifting of $\psi$ :

$$
\begin{array}{ccccccc}
\mathscr{A} & \rightarrow & \mathscr{T}^{0} & \rightarrow & \mathscr{T}^{1} & \rightarrow \cdots \\
\downarrow \psi & & \downarrow \psi^{0} & & \downarrow \psi^{1} & \\
f^{*} \mathscr{A} & \rightarrow & \hat{T}^{0} & \rightarrow & \hat{T}^{1} & \rightarrow \cdots
\end{array}
$$

Application of the $\mathbf{g}$ functor leads, via 1.1(e), to the following commutative diagram:

$$
\begin{array}{cccccc}
\mathbf{g}(\mathscr{A}) & \rightarrow & \mathbf{g}\left(\mathscr{T}^{0}\right) & \rightarrow & \mathbf{g}\left(\mathscr{T}^{1}\right) & \rightarrow \cdots \\
\downarrow \mathbf{g}(\psi) & & \downarrow \mathbf{g}\left(\psi^{0}\right) & & \downarrow \mathbf{g}\left(\psi^{1}\right) & \\
\mathbf{g}\left(f^{*} \mathscr{A}\right) \rightarrow \mathbf{g}\left(\hat{T}^{0}\right) & \rightarrow & \mathbf{g}\left(\hat{T}^{1}\right) & \rightarrow \cdots
\end{array}
$$

The resolutions, (1.3.1), of the chains in (1.5.3) can be chosen to lift the chain map $\mathbf{g}\left(\psi^{*}\right)$. Accordingly, there are sheaf homomorphisms $\lambda^{p q}: \mathscr{B}^{p q} \rightarrow \hat{\mathscr{B}}^{p q}$ which commute with the $d$-homomorphisms. Then

$$
\Gamma\left(\lambda^{p q}\right): \Gamma\left(T, \mathscr{B}^{p q}\right) \rightarrow \Gamma\left(T, \hat{\mathscr{B}}^{p q}\right) .
$$

There result spectral sequence homomorphisms ${ }_{1} \lambda_{r}^{p q}:{ }_{1} E_{r}^{p q} \rightarrow{ }_{1} \hat{E}_{r}^{p q}$ and ${ }_{2} \lambda_{r}^{p q}:{ }_{2} E_{r}^{p q}$ $\rightarrow{ }_{2} \hat{E}_{r}^{p q}$.

The corresponding homomorphism of the targets constitutes (1.5.2). Indeed the chain map ${ }_{1} \lambda_{1}^{* 0}:{ }_{1} E_{1}^{* 0} \rightarrow{ }_{1} \hat{E}_{1}^{* 0}$ may be represented, in view of (1.3.3), by:

$$
\begin{array}{cccc}
\Gamma\left(T, \mathbf{g}\left(\mathscr{T}^{0}\right)\right) & \rightarrow & \Gamma\left(T, \mathbf{g}\left(\mathscr{T}^{1}\right)\right) & \rightarrow \cdots \\
\downarrow\left(\mathbf{g}\left(\psi^{0}\right)\right) & & \downarrow \Gamma\left(\mathbf{g}\left(\psi^{1}\right)\right) & \\
\Gamma\left(T, \mathbf{g}\left(\hat{\mathscr{T}}^{0}\right)\right) & \rightarrow & \Gamma\left(T, \mathbf{g} \mathbf{f}\left(\hat{\mathscr{T}}^{1}\right)\right) & \rightarrow \cdots
\end{array}
$$


The identifications $\Gamma\left(T, \mathbf{g}\left(\mathscr{T}^{0}\right)\right)=\Gamma\left(Y, \mathscr{T}^{p}\right)$ and $\Gamma\left(T, \mathbf{g f}\left(\hat{\mathscr{T}}^{p}\right)\right)=\Gamma\left(X, \hat{\mathscr{T}}^{p}\right)$, together with 1.1(e) and (f) culminate in:

$$
\begin{array}{cccc}
\Gamma\left(Y, \mathscr{T}^{p}\right) & \rightarrow & \Gamma\left(Y, \mathscr{T}^{1}\right) & \rightarrow \cdots \\
\downarrow \Gamma\left(\psi^{0}\right) & & \downarrow \Gamma\left(\psi^{1}\right) & \\
\Gamma\left(X, \hat{\mathscr{T}}^{0}\right) & \rightarrow & \Gamma\left(X, \hat{\mathscr{T}}{ }^{1}\right) & \rightarrow \cdots
\end{array}
$$

Application of the cohomology function to (1.5.4) yields (1.5.2).

Next we determine the homomorphisms ${ }_{2} \lambda_{2}^{p q}:{ }_{2} E_{2}^{p q} \rightarrow{ }_{2} \hat{E}_{2}^{p q}$. The homomorphisms $\Gamma\left(\lambda^{p q}\right): \Gamma\left(T, \mathscr{B}^{p q}\right) \rightarrow \Gamma\left(T, \hat{\mathscr{B}}^{p q}\right)$ induce homomorphisms

$$
{ }_{1} H\left(\Gamma\left(\lambda^{* p}\right)\right):{ }_{1} H^{q}\left(\Gamma\left(T, \mathscr{B}^{* p}\right)\right) \rightarrow{ }_{1} H^{q}(\Gamma(T, \hat{\mathscr{B}} * p))
$$

which commute with the induced homomorphisms ${ }_{1} H\left({ }_{2} d\right)$ and ${ }_{1} H\left({ }_{2} \hat{d}\right)$. On taking account of the identifications of (1.3.7),

$$
\Gamma\left(H\left(\lambda^{* p}\right)\right): \Gamma\left(T, H^{q}(\mathscr{B} * p)\right) \rightarrow \Gamma\left(T, H^{q}\left(\hat{\mathscr{B}}^{* p}\right)\right) .
$$

Application of the cohomology functor along the columns of the resolutions (1.3.1) results in

$$
\begin{aligned}
& \mathscr{H}^{q}(\mathbf{g}, \mathscr{A})=H^{q}\left(\mathbf{g}\left(\mathscr{T}^{*}\right)\right) \quad \rightarrow \quad{ }_{1} H^{q}\left(\mathscr{B}^{* 0}\right) \stackrel{{ }_{1}^{H\left({ }_{2} d\right)}}{\rightarrow}{ }_{1} H^{q}\left(\mathscr{B}^{* 1}\right) \rightarrow \cdots \\
& \downarrow \mathbf{g}(\psi)^{*} \quad \downarrow H\left(\mathbf{g}\left(\psi^{*}\right)\right) \quad \downarrow_{1} H\left(\lambda^{* 0}\right) \quad \downarrow_{1} H\left(\lambda^{* 1}\right) \\
& \mathscr{H}^{q}\left(\mathbf{g f}, f^{*} \mathscr{A}\right)=H^{q}\left(\mathbf{g f}\left(\hat{\mathscr{T}}^{*}\right)\right) \rightarrow{ }_{1} H^{q}\left(\hat{\mathscr{B}}^{* 0}\right) \stackrel{{ }_{1} H\left({ }_{2} d\right)}{\rightarrow}{ }_{1} H^{q}\left(\hat{\mathscr{B}}^{* 1}\right) \rightarrow \cdots
\end{aligned}
$$

where the sheaf homomorphism $\mathbf{g}(\psi)^{*}$ comes from (1.5.3). In view of (1.3.6),

$$
\begin{array}{ccc}
{ }_{2} E_{2}^{p q} & = & H^{p}\left(T, \mathscr{H}^{q}(\mathbf{g}, \mathscr{A})\right) \\
\downarrow_{2} \lambda_{2}^{p q} & & \downarrow H\left(\mathbf{g}(\psi)^{*}\right) \\
{ }_{2} \hat{E}_{2}^{p q} & = & H^{p}\left(T, \mathscr{H}^{q}\left(\mathbf{g f}, f^{*} \mathscr{A}\right)\right)
\end{array}
$$

1.6. We follow the conventional usage of $\left\{E_{r}^{p q}, E^{*}\right\}$ for a spectral sequence and its target. Similarly, $\left\{\hat{E}_{r}^{p q}, \hat{E}^{*}\right\}$. If the homomorphisms $\lambda_{r}^{p q}: E_{r}^{p q} \rightarrow \hat{E}_{r}^{p q}$ commute with the $d_{r}$ homomorphisms and are compatible with the identifications

$$
H\left(E_{r}^{p q}\right)=E_{r+1}^{p q}, \quad H\left(\hat{E}_{r}^{p q}\right)=\hat{E}_{r+1}^{p q},
$$

then $\left\{\lambda_{r}^{p q}\right\}$ is a homomorphism of spectral sequences. They induce a homomorphism $\lambda_{\infty}^{p q}: E_{\infty}^{p q} \rightarrow \hat{E}_{\infty}^{p q}$. Let $\psi^{*}: E^{*} \rightarrow \hat{E}^{*}$ be a filtration preserving homomorphism compatible with $\lambda_{\infty}$, in the sense of the following:

$$
\begin{array}{ccc}
E_{\infty}^{p q} & \approx & F_{p} E^{p+q} / F_{p+1} E^{p+q} \\
\downarrow \lambda_{\infty}^{p q} & & \downarrow \tilde{\psi}_{p}^{p+q} \\
\hat{E}_{\infty}^{p q} & \approx & F_{p} \hat{E}^{p+q} / F_{p+1} \hat{E}^{p+q}
\end{array}
$$

Write $\left\{\lambda_{r}^{p q}, \psi^{*}\right\}:\left\{E_{r}^{p q}, E^{*}\right\} \rightarrow\left\{\hat{E}_{r}^{p q}, \hat{E}^{*}\right\}$ for such a pair.

Our conclusions are summarized in

THEOREM 1.7. Suppose $f: X \rightarrow Y$ is a continuous surjection. Let $\mathbf{g}: \mathbb{S}(Y) \rightarrow \subseteq(T)$ and $\mathbf{g f :} \Xi(X) \rightarrow \Xi(T)$ be functors satisfying 1.1(a)-(f). Then for any arbitrary sheaf 
$\mathscr{A}$ on $Y$ there are spectral sequences $E_{r}^{p q}$ and $\hat{E}_{r}^{p q}$ with targets $H^{*}(Y, \mathscr{A})$ and $H^{*}\left(X, f^{*} \mathscr{A}\right)$ respectively whose initial terms are

$$
E_{2}^{p q}=H^{p}\left(T, \mathscr{H}^{q}(\mathbf{g}, \mathscr{A})\right)
$$

and

$$
\hat{E}_{2}^{p q}=H^{p}\left(T, \mathscr{H}^{q}\left(\mathbf{g f}, f^{*} \mathscr{A}\right)\right) .
$$

Futhermore, there is a homomorphism of spectral sequences $\lambda_{r}^{p q}: E_{r}^{p q} \rightarrow \hat{E}_{r}^{p q}$ satisfying

$$
\left\{\lambda_{r}^{p q}, \psi^{*}\right\}:\left\{E_{r}^{p q}, H^{*}(Y, \mathscr{A})\right\} \rightarrow\left\{\hat{E}_{r}^{p q}, H^{*}\left(X, f^{*} \mathscr{A}\right)\right\}
$$

and

(1.7)(d) $\lambda_{2}^{p q}=H\left(\mathbf{g}(\psi)^{*}\right): H^{p}\left(T, \mathscr{H}^{q}(\mathbf{g}, \mathscr{A})\right) \rightarrow H^{p}\left(T, \mathscr{H}^{q}\left(\mathbf{g f}, f^{*} \mathscr{A}\right)\right)$,

where the sheaf homomorphism $g(\psi)^{*}$ arises from (1.5.3).

Since our resolutions start with $p=0, q=0$,

$$
E^{p q}=\hat{E}^{p q}=0 \text { if } p<0 \text { or } q<0 .
$$

2. The topology of $T$. Consider a set-valued function $g$ on $Y$ onto $T$. Let $G$ be the inverse of $g$ and denote the set $g^{-1}(t)=G(t)$ by $G_{t}$. Then $G(T)=g^{-1}(T)=Y$, $g(Y)=T$, and $\left\{G_{t} \mid t \in T\right\}$ is a covering of $Y$ for which $G_{t} \neq \varnothing$.

Conversely, if $\left\{G_{t} \mid t \in T\right\}$ is a covering of $Y$ by not necessarily distinct sets and no $G_{t}$ is empty, then the function $g$ defined by $g(y)=\left\{t \mid y \in G_{t}\right\}$ is a set-valued function where $g(Y)=T$ and $g^{-1}(T)=Y$, by virtue of the observation that $G_{t} \neq \varnothing$ and that the sets $G_{t}$ cover $Y$. Furthermore, $g^{-1}(t)=G(t)=G_{t}$.

A subset $B$ of $Y$ is saturated if $B=\cup_{t \in S} G_{t}$ for $S \subset T$; or equivalently, for each $y \in B$, some $G_{t} \subset B$ contains $y$.

Our first concern is with sufficient conditions for which the relation $g$ determines a functor $\mathbf{g}$ of the type considered in $\S 1$. Whether or not $T$ is topologized initially, $g$ is used to determine the topology on $T$ used for the sheaves $\mathbf{g}(\mathscr{A})$. Unfortunately, this topology will not be Hausdorff in general.

Definition 2.1. A collection $\left\{G_{t} \mid t \in T\right\}$ of subsets of $Y$ is a determining collection provided:

2.1(a) The collection is a covering of $Y$ by nonempty, closed sets.

2.1(b) For every $t_{1}$ and $t_{2}, G_{t_{1}} \cap G_{t_{2}}$ is saturated.

2.1(c) If $V$ is any open neighborhood of $G_{t}$, there is an open saturated set $W$ for which $G_{t} \subset W \subset V$.

From the point of view of the relation $g$, these conditions are equivalent to:

2.1( $\left.\mathrm{a}^{\prime}\right) G(T)=Y, g(Y)=T$ and $G(t)$ is closed for each $t \in T$.

2.1(b') $G\left(t_{1}\right) \cap G\left(t_{2}\right)=G(S)$ for some $S \subset T$.

2.1( $\left.\mathrm{c}^{\prime}\right)$ Every open neighborhood of $G(t)$ contains an open neighborhood of the form $W=G(S)$, of $G(t)$.

Assume for the remainder of this section that $\left\{G_{t} \mid t \in T\right\}$ is a determining collection.

Lemma 2.2. For $B \subset Y, g(B)=\left\{t \mid G_{t} \cap B \neq \varnothing\right\}$. 
LEMMA 2.3. For $B \subset Y$, the complement of $B, B^{\tilde{y}}$, contains $G(g(B) \tilde{)})$.

Moreover, $B^{\tilde{~}}$ is saturated if and only if $\boldsymbol{B}^{\tilde{B}}=G(g(B) \tilde{)})$.

If $y \in G(g(B) \tilde{)})$, then $y \in G_{t}$ for some $t \notin g(B)$. By 2.2, $G_{t} \cap B=\varnothing$ and so $y \in G_{t} \subset B^{\tilde{B}}$. Thus $G(g(B) \tilde{)}) \subset B^{\tilde{\nu}}$.

For the second part of 2.3, suppose $B^{\sim}$ is saturated. Then $y \in B^{\sim}$ implies $y \in G_{t} \subset B^{\tilde{y}}$, where $t \in g(B)^{\sim}$ by 2.2. Accordingly, $y \in G_{t} \subset G\left(g(B)^{\tilde{\gamma})}\right.$ ) and so $B^{\sim} \subset G(g(B) \tilde{)})$.

Conversely, if

$$
\tilde{B}=G\left(g(B)^{\tilde{T}}\right)=\bigcup\left\{G_{t} \mid t \in g(B) \tilde{}\right\}
$$

then $B^{\sim}$ is saturated.

Denote by $\Omega$ the collection of all closed sets $B$ in $Y$ with saturated complements and let

$$
g(\Re)=\{g(B) \mid B \in \Re\} .
$$

LEMMA 2.4. $\Re$ and $g(\Re)$ are closed under finite unions.

For $B_{1}$ and $B_{2}$ in $\Re$, suppose $y \in\left(B_{1} \cup B_{2}\right) \tilde{\text {. Since }} B_{1}^{\sim}$ and $B_{2}^{\sim}$ are saturated,

$$
y \in G_{t_{1}} \cap G_{t_{2}} \subset \tilde{B_{1}} \cap \tilde{B_{2}}
$$

for some $t_{1}$ and $t_{2}$ in $T$. According to 2.1(b)

$$
y \in G_{t} \subset G_{t_{1}} \cap G_{t_{2}} \subset \tilde{B_{1}} \cap \tilde{B_{2}}
$$

for some $t \in T$. Therefore $\left(B_{1} \cup B_{2}\right)$ is saturated; that is to say $B_{1} \cup B_{2}$ is in $\Omega$. Moreover, $g\left(B_{1} \cup B_{2}\right)=g\left(B_{1}\right) \cup g\left(B_{2}\right)$ is in $g(\Omega)$.

Thus, $g(\Re)$ is a basis for the closed sets of a topology $\mathfrak{I}_{\mathfrak{g}}$ on $T$. Let $\mathfrak{I}_{g}$ denote the topology determined by the basis $\mathfrak{B}_{g}=\{U \mid \tilde{U} \in g(\Re)\}$.

LEMMA 2.5. (a). If $V \in \mathfrak{B}_{g}$ and $G_{t} \subset G(V)$, then $t \in V$.

(b) If $V_{1}$ and $V_{2}$ are open subsets of $T$, then $G\left(V_{1} \cap V_{2}\right)=G\left(V_{1}\right) \cap G\left(V_{2}\right)$.

(c) $G(U)$ is open whenever $U$ is open.

(d) For each fixed $t,\left\{G(U) \mid t \in U \in \mathfrak{I}_{g}\right\}$ constitutes an open neighborhood basis of $G_{t}$.

$\operatorname{Ad}(\mathrm{a})$. Let $V=g(B) \tilde{\text { in }} \mathfrak{B}_{g}$. Then $G(V)=B^{\sim}$ by 2.3 , whence $G_{t} \cap B=\varnothing$. That $t \in V$ follows from 2.2 .

Ad(b). Plainly $G\left(V_{1} \cap V_{2}\right)$ is contained in $G\left(V_{1}\right) \cap G\left(V_{2}\right)$ for any subset pair in $T$. Let $y \in G\left(V_{1}\right) \cap G\left(V_{2}\right)$ for $V_{1}$ and $V_{2}$ in $\mathfrak{T}_{g}$. Choose $t_{i}$ in $V_{i}, i=1,2$, to satisfy $y \in G_{t_{i}} \subset G\left(V_{i}\right)$. Then

$$
t_{i} \in U_{i} \subset V_{i} \text { for } U_{i} \in \mathfrak{B}_{g} .
$$

Since $G_{t_{1}} \cap G_{t_{2}}$ is saturated, $y \in G_{t} \subset G_{t_{1}} \cap G_{t_{2}}$ for some $t$. Therefore, $G_{t} \subset G_{t_{i}} \subset$ $G\left(U_{i}\right)$ and consequently, $t \in U_{1} \cap U_{2}$ by (a). Hence

$$
y \in G_{t} \subset G\left(U_{1} \cap U_{2}\right) \subset G\left(V_{1} \cap V_{2}\right) .
$$

$\operatorname{Ad}(\mathrm{c})$. For $U \in \mathfrak{I}_{g}, U=\bigcup V_{a}$ where $V_{a} \in \mathfrak{B}_{g}$. Since $G(U)=\bigcup G\left(V_{a}\right)$, it suffices to show that $G(U)$ is open for $U \in \mathfrak{B}_{g}$. Let $B \in \Re$ satisfy $g(B)=\tilde{U^{\alpha}}$. Since $\boldsymbol{B}^{\sim}$ is saturated, $G(U)=B^{\sim}$ an open set. 
Ad(d). For $t \in U \in \mathfrak{I}_{g}, G(U)$ is an open neighborhood of $G_{t}$. Let $V$ be an arbitrary open neighborhood of $G_{t}$ in $Y$ and let $W$ be an open saturated neighborhood of $G_{t}$ contained in $V$. Then $B=\tilde{W} \in \Re$, whence $U=g(B)^{\tilde{y}}$ is in $\mathfrak{B}_{g}$. According to 2.3

$$
G(U)=G\left(g(B)^{\sim}\right)=W .
$$

Hence $G_{t} \subset G(U) \subset V$ and $t \in U$ by (a).

An immediate consequence of $2.5(\mathrm{~d})$ is

Corollary 2.6. $G_{t}=\bigcap\left\{G(U) \mid t \in U \in \mathfrak{I}_{g}\right\}$.

3. Some examples. The role of $2.1(\mathrm{a})-(\mathrm{c})$ and the topology $\mathfrak{I}_{g}$ is brought into focus by some illustrative examples.

EXAMPLE 3.1. Let $g: Y \rightarrow T$ be a (single valued) function assumed closed, continuous and onto. Then $\left\{G_{t}=g^{-1}(t) \mid t \in T\right\}$ is a covering of $Y$ by pairwise disjoint closed sets. Suppose $V$ is an open neighborhood of $g^{-1}(t)$.

$$
W=g^{-1}\left(g(\tilde{V})^{\sim}\right)=G(g(\tilde{V}) \sim)
$$

is an open saturated set, since $g$ is closed and continuous. Evidently, $g^{-1}(t) \in W \subset V$.

Here, $\mathfrak{I}_{g}$ coincides with the original topology $\mathfrak{I}$ of $T$. Indeed, $g(\Re)$ is contained in the collection of closed sets in the topology $\mathfrak{I}$. Hence $\mathfrak{I}_{g} \subset \mathfrak{I}$. On the other hand if $K$ is closed in $(T, \mathfrak{I})$, then $B=g^{-1}(K)$ is a closed subset of $Y$. Since $G(\tilde{K})$ $=g^{-1}(\tilde{K})=\tilde{B}, \tilde{B}$ is saturated and so $g(B)$ is closed in $\left(T, \mathfrak{I}_{g}\right)$. Since $g$ is onto, $g(B)=g\left(g^{-1}(K)\right)=K$ and consequently $\mathfrak{I} \subset \mathfrak{I}_{g}$.

EXAMPLE 3.2. Supppose $\left\{G_{t} \mid t \in T\right\}$ is a partition of $Y$ by nonempty closed sets such that when $B$ is closed

$$
\bigcup\left\{G_{t} \mid G_{t} \cap B \neq \varnothing\right\} \text { is closed. }
$$

Then $T$ may be considered the quotient space of the equivalence relation determined by the partition. If $g: Y \rightarrow T$ is the identification map, then by reason of (3.2.1) $g$ is closed. By Example 3.1, $\left\{G_{t} \mid t \in T\right\}$ is a determining set and so $\mathfrak{I}_{g}$ is merely the identification topology on $T$.

EXAMPLE 3.3. If $g: Y \rightarrow T$ is a relation satisfying $2.1\left(\mathrm{a}^{\prime}\right), 2.1\left(\mathrm{~b}^{\prime}\right)$, and

$$
G\left(g(B)^{\sim}\right) \text { is open whenever } B \text { is closed, }
$$

then $g$ also satisfies 2.1( $\left.\mathrm{c}^{\prime}\right)$. Indeed, if $V$ is an open neighborhood of $G_{t}$ and $B=\tilde{V}$, then $G(g(B) \tilde{)})$ is an open, saturated neighborhood of $G_{t}$ contained in $V$.

EXAMPLE 3.4. If $H$ is a compact subgroup of the multiplicative group $Y$, the right cosets of $H,\{H \cdot y \mid y \in Y\}$, constitute a determining collection. Conditions 2.1(a) and (b) are clearly satisfied. To establish 2.1(c), let $H \cdot y$ be contained in the open set $V$. Write $m\left(y_{1}, y_{2}\right)$ for the product $y_{1} \cdot y_{2}$. Then $H \times y \subset m^{-1}(V)$, where $m^{-1}(V)$ is open in $Y \times Y$. The compactness of $H$ implies that for some open $W$ containing $y$, $H \times W \subset m^{-1}(V)$. Hence

$$
H \cdot y \subset H \cdot W=m(H \times W) \subset V,
$$

where

$$
H \cdot W=\bigcup_{y \in H} y \cdot W=\bigcup_{y \in W} H \cdot y
$$


is both open and saturated. Accordingly, the collection of right cosets satisfies 2.1(c). In this example we can identify $T$ with either $Y$ or the quotient group $Y / H$. In the latter case, $\mathfrak{I}_{g}$ is the identification topology.

We show that the compactness condition on $H$ cannot be dropped in general in Example 3.4.

EXAMPLE 3.5. Let $Y=R^{1}$, the additive group of reals, and let $H=J$ be the subgroup of integers. For $\varepsilon_{n}=1 /(|n|+2)$, let $V$ be the union of open intervals

$$
V=\bigcup_{n \in J}\left(n-\varepsilon_{n}, n+\varepsilon_{n}\right)
$$

Then, $V$ is an open neighborhood of $J$ which is free of open saturated subsets, and it is easy to see that $J$ is the only right coset contained in $V$.

The next example provides a general scheme for generating a determining collection. From this a collection for $Y \subset R^{n}$ is obtained by taking $T \subset R^{1}, d(y)=\|y\|$, the norm of $y$, and $h(t)=t$.

EXAMPLE 3.6. Suppose now that $T$ is merely an untopoplogized set, that $d$ : $Y \rightarrow R^{1}$ is continuous and closed, and that $h: T \rightarrow R^{1}$ is a function, subject to the following conditions:

(3.6.1) If $d(y)<r$, then for some $t \in T, d(y) \leqslant h(t)<r$.

(3.6.2) If $t \in T$, some $y \in Y$ satisfies $d(y) \leqslant h(t)$.

For $t \in T$, let

(3.6.3) $G_{t}=\{y \mid d(y) \leqslant h(t)\}=d^{-1}((-\infty, h(t)])$.

Then:

(3.6.4) Each $G_{t}$ is closed since $d$ is continuous.

(3.6.5) $G_{t} \neq \varnothing$ by (3.6.2).

(3.6.6) $\cup G_{t}=Y$ by (3.6.1).

(3.6.7) $G_{t_{1}} \cap G_{t_{2}}$ is saturated since $h(t) \leqslant h\left(t^{\prime}\right)$ implies $G_{t} \subset G_{t^{\prime}}$.

(3.6.8) If $U$ is an open neighborhood of $G_{t}$, there is a saturated, open set $W$ with $G_{t} \subset W \subset U$.

To verify (3.6.8), let $G_{t}$ be in the open set $U$. By (3.6.3), $d(y)>h(t)$ for $y \in$ $\tilde{U^{\sim}} \subset G_{t}$. Hence $(-\infty, h(t)] \cap d\left(\tilde{U^{\tilde{N}}}\right)=\varnothing$. Since $d$ is a closed map, $d\left(\tilde{U^{2}}\right)$ is closed and consequently

(3.6.9) $(-\infty, h(t)+\varepsilon) \cap d\left(\tilde{U^{\tau}}\right)=\varnothing$ for some positive $\varepsilon$.

Then,

$$
W=\bigcup\left\{G_{s} \mid h(s)<h(t)+\varepsilon\right\}
$$

is a saturated subset of $Y$ containing $G_{t}$.

If $y \in W$, then $y \in G_{s}$ for some $s$ for which $h(s)<h(t)+\varepsilon$. Then $d(y) \leqslant h(s)<$ $h(t)+\varepsilon$ and so $d(y) \notin d(\tilde{U})$ by (3.6.9). Accordingly, $y \in U$ and thus $W \subset U$.

Again for $y \in W, d(y) \in(-\infty, h(t)+\varepsilon)$. Since $d$ is continuous,

$$
d\left(N_{y}\right) \subset(-\infty, h(t)+\varepsilon)
$$

for a neighborhood $N_{y}$ of $y$. If $z \in N_{y}, d(z)<h(t)+\varepsilon$ and so by (3.6.1)

$$
d(z) \leqslant h(s)<h(t)+\varepsilon
$$

for an $s$ in $T$. Consequently, $z \in G_{s} \subset W$ whence $N_{y} \subset W$. Therefore, $W$ is open, which is the assertion of (3.6.8). 
The validity of (3.6.4)-(3.6.8) implies that $\left\{G_{t} \mid t \in T\right\}$ is a determining collection of sets in $Y$.

The assumption that $d$ is closed may be replaced by

(3.6.10) If $d^{-1}(h(t)) \subset U=$ an open set, there is a positive $\varepsilon$ with

$$
d^{-1}([h(t), h(t)+\varepsilon)) \subset U .
$$

In this case the proof of (3.6.8) must be modified. Indeed, for $G_{t} \subset U, d^{-1}(h(t)) \subset U$ by (3.6.3). Choose $\varepsilon>0$ to satisfy (3.6.10). Then

$$
\begin{aligned}
d^{-1}((-\infty, h(t)+\varepsilon)) & =d^{-1}((-\infty, h(t)]) \cup d^{-1}([h(t), h(t)+\varepsilon)) \\
& =G_{t} \cup d^{-1}([h(t), h(t)+\varepsilon)) \subset U
\end{aligned}
$$

Again, let

$$
W=\bigcup\left\{G_{s} \mid h(s)<h(t)+\varepsilon\right\},
$$

which is a saturated, open neighborhood of $G_{t}$. For $y \in G_{s}$ with $h(s)<h(t)+\varepsilon$, $d(y) \leqslant h(s)<h(t)+\varepsilon$. Therefore, $y \in d^{-1}((-\infty, h(t)+\varepsilon))$ and so $W \subset U$.

Next we establish:

(3.6.11) The collection of sets $\left\{h^{-1}((-\infty, r)) \mid r \in R^{1} \cup\{ \pm \infty\}\right\}$ contains the topology $\mathfrak{I}_{g}$.

For $-\infty \leqslant r \leqslant \infty$, let

$$
U_{r}=\bigcup\left\{G_{s} \mid h(s)<r\right\},
$$

where $U_{\infty}=Y$ and $U_{-\infty}=\varnothing$. The argument used for proving (3.6.8) demonstrates that $U_{r}$ is an open saturated set.

In fact, every open saturated set is of this form. For an open saturated set $U$, let

(3.6.12) $r=\sup \left\{p \mid h(s)<p\right.$ implies $\left.G_{s} \subset U\right\}$.

If the set on the right-hand side is empty, put $r=-\infty$; if there is no upper bound, set $r=\infty$. If $h(s)<r, h(s)<p \leqslant r$ for some $p$, and therefore $G_{s} \subset U$. Consequently

$$
U_{r}=\bigcup\left\{G_{s} \mid h(s)<r\right\} \subset U .
$$

For $y \in U, y \in G_{t} \subset U$ for some $t \in T$ because $U$ is saturated. Again by the argument for (3.6.8), for some $\varepsilon>0, h(s)<h(t)+\varepsilon$ implies $G_{s} \subset U$. For $p=h(t)$ $+\varepsilon, h(t)<p \leqslant r$ and so $y \in G_{t} \subset U_{r}$. Hence $U \subset U_{r}$. Therefore $U=U_{r}$.

Let $U$ be an open saturated set and let $r$ be defined by (3.6.12). For $B=\tilde{U \text {, }}$

$$
g(B)^{\sim}=\left\{t \mid G_{t} \subset U\right\}
$$

by Lemma 2.2. Since $h(t)<r$ implies $G_{t} \subset U_{r}=U$,

$$
h^{-1}((-\infty, r)) \subset g(B)^{\sim}
$$

For $t \in g(B)^{\tilde{}}, G_{t} \subset U$ which implies $h(t)<r$. Therefore, $t \in h^{-1}((-\infty, r))$. Consequently, $g(B) \tilde{})^{-1}((-\infty, r))$.

Hence there is a subset $S$ of $R \cup\{ \pm \infty\}$ for which

$$
\mathfrak{B}_{g}=\left\{h^{-1}((-\infty, r)) \mid r \in S\right\},
$$

where $\mathfrak{B}_{g}$ is the basis for $\mathfrak{I}_{g}$. This collection is contained in

$$
\left\{h^{-1}((-\infty, r)) \mid r \in R \cup\{ \pm \infty\}\right\},
$$

and is closed under arbitrary unions and therefore includes $\mathfrak{I}_{g}$. 
In general (3.6.11) cannot be strengthened to set equality. The difficulty is that for some $r^{1}$ it is possible that $h\left(r^{1}\right)<h(r)$ and yet no $y \in Y$ exists for which $h\left(r^{1}\right) \leqslant$ $d(y)<h(r)$. In such a case $U_{r^{1}}=U_{r}$ and so $h^{-1}\left(\left(-\infty, r^{1}\right)\right)$ may not be in $\mathfrak{I}_{g}$.

EXAMPLE 3.7. For $Y$ closed in $R^{n}$, let $d: Y \rightarrow R^{1}$ be the restriction of the norm function on $R^{n}$ to $Y$, i.e. $d(y)=\|y\|$. Since $\|\cdot\|: R^{n} \rightarrow R^{1}$ is continuous and closed and $Y$ is closed, $d$ is continuous and closed. For $a=\inf \{\|y\| \mid y \in Y\}$, let $T=[a, \infty)$ and write $h: T \rightarrow R^{1}$ for the inclusion map. Since $d(Y)$ is closed in $R, a=d\left(y_{1}\right)$ for some $y_{1} \in Y$.

If $d(y)=\|y\|<r, d(y) \in T$ and so $d(y)=h(d(y))<r$. For $t \in T, d\left(y_{1}\right)=a$ $\leqslant t=h(t)$. Therefore, conditions (3.6.1) and (3.6.2) are satisfied, accordingly (3.6.3) defines a determining collection of sets in $Y$. For $r>0$, let

$$
B_{r}=\left\{y \in R^{n} \mid\|y\| \leqslant r\right\}, \quad S_{r}=\left\{y \in R^{n} \mid\|y\|=r\right\}, \quad N_{r}=\left\{y \in R^{n} \mid\|y\|<r\right\} .
$$

Then

$$
G_{t}=\left\{y \in Y^{n} \mid\|y\| \leqslant h(t)=t\right\}=B_{t} \cap Y .
$$

Hence, if

$$
Y=R^{n}-N_{\varepsilon}=\left\{y \in R^{n} \mid\|y\| \geqslant \varepsilon\right\},
$$

then $T=[\varepsilon, \infty), G_{t}=\{y \mid \varepsilon \leqslant\|y\| \leqslant t\}=B_{t}-N_{\varepsilon}$ and $G_{\varepsilon}=S_{\varepsilon}$. Consequently, the saturated open sets will be the sets $U_{r}=\{y \mid \varepsilon \leqslant\|y\|<r\}$ and the topology on $T$ is $\mathfrak{I}_{g}=\{[\varepsilon, r) \mid \varepsilon<r \leqslant \infty\}$.

EXAMPLE 3.8. If in Example $3.7 Y=R^{n}-\{0\}, Y$ is no longer closed and neither is $d$. However, (3.6.10) is satisfied since if $U$ is an open neighborhood of $d^{-1}(t)=S_{t}$, there is an $\varepsilon>0$ satisfying

$$
d^{-1}([t, t+\varepsilon))=\{y \mid t \leqslant\|y\|<t+\varepsilon\} \subset U .
$$

In this case

$$
G_{t}=B_{t}-\{0\}=\{y \mid 0<\|y\| \leqslant t\}
$$

and the induced topology on $T$ consists of the open sets $\{(0, t) \mid 0<t \leqslant \infty\}$.

4. The two map induced functors. Our way of obtaining functors that satisfy the abstract conditions of 1.1 is by use of $\mathfrak{I}_{g}$. Thus, we introduce map induced functors $\mathrm{g}$ and gf satisfying 1.1 (which explains our use of the same notation, $\mathbf{g}$ ). $f: X \rightarrow Y$ will invariably be a continuous, closed surjection of paracompact spaces while $\left\{G_{t} \mid t \in T\right\}$ is a determining collection in $Y$ and $T$ is endowed with the topology $\mathfrak{T}_{g}$. Support families of closed supports are used throughout.

A presheaf over $T$ for $\mathscr{A} \in \mathscr{S}(Y)$ is defined by

$$
U \rightarrow \Gamma(G(U), \mathscr{A}) \text {. }
$$

For $V \subset U$, the restriction $r_{V}^{U}$ of a section $s$ in $\Gamma(G(U), \mathscr{A})$ is the section $s \mid G(V)$ in $\Gamma(G(V), \mathscr{A})$.

We assert that

(4.1.2) The presheaf (4.1.1) is a sheaf on $T$, denoted by $\mathrm{g}(\mathscr{A})$, with 
(4.1.2)(a)

$$
\Gamma(U, \mathbf{g}(\mathscr{A}))=\Gamma(G(U), \mathscr{A}) \text { for } U \in \mathfrak{T}_{g} .
$$

Let $U=\bigcup U_{a}$ for sets in $\mathfrak{I}_{g}$. If $s \in \Gamma(G(U), \mathscr{A})$ where $s \mid G\left(U_{a}\right)=0$ : for each $a$, then $s=0$ since $G(U)=G\left(\cup U_{a}\right)=\bigcup G\left(U_{a}\right)$.

Assume for any arbitrary index, say $a, s_{a} \in \Gamma\left(G\left(U_{a}\right), \mathscr{A}\right)$ subject to

$$
s_{a}\left|G\left(U_{a} \cap U_{b}\right)=s_{b}\right| G\left(U_{a} \cap U_{b}\right) .
$$

For $y \in G(U), y \in G\left(U_{a}\right)$ for some $a$. Write $s(y)=s_{a}(y)$. If $y \in G\left(U_{b}\right)$ also, then

$$
y \in G\left(U_{a}\right) \cap G\left(U_{b}\right)=G\left(U_{a} \cap U_{b}\right)
$$

(see 2.5(b)). Thus, $s_{a}(y)=s_{b}(y)$ and, accordingly, $s$ is well defined. Since $\left\{G\left(U_{a}\right)\right\}$ is an open cover of $G(U), s \in \Gamma(G(U), \mathscr{A})$ with $s \mid G\left(U_{a}\right)=s_{a}$.

Suppose $\alpha: \mathscr{A} \rightarrow \mathscr{B}$ is a sheaf homomorphism. For $s_{U} \in \Gamma(G(U), \mathscr{A})$, the composition $\alpha s_{U}$ is a section in $\Gamma(G(U), \mathscr{B})$. Denote by $\alpha_{U}$ the correspondence $\Gamma(G(U), \mathscr{A}) \rightarrow \Gamma(G(U), \mathscr{B})$ so defined. Clearly, $\left\{\alpha_{U} \mid U \in \mathfrak{I}_{g}\right\}$ represents a presheaf homomorphism. Consequently, $\alpha$ induces a sheaf homomorphism $\mathbf{g}(\alpha): \mathbf{g}(\mathscr{A})$ $\rightarrow \mathrm{g}(\mathscr{B})$. Thus, $\mathbf{g}$ is a functor from $\mathfrak{S}(Y)$ to $\mathfrak{S}(T)$ whose properties are made up of 1.1(a)-(c), according to

Proposition 4.2. (a) $\mathrm{g}$ is an additive, left exact covariant functor.

(b) $\mathbf{g}(\mathscr{A})$ is flabby whenever $\mathscr{A}$ is flabby.

(c) There is a natural equivalence $\Gamma(Y, \cdot) \stackrel{\approx}{\rightarrow} \Gamma(T, \mathbf{g}(\cdot))$.

Ad(a). Additivity and covariance are well-known consequences of the definition of $g(\alpha)$. The left exactness is a consequence of (4.1.2)(a). Indeed, if

$$
0 \rightarrow \mathscr{A}_{1} \stackrel{\alpha}{\rightarrow} \mathscr{A} \stackrel{\beta}{\rightarrow} \mathscr{A}_{2}
$$

is exact, so is

$$
0 \rightarrow \Gamma\left(G(U), \mathscr{A}_{1}\right) \stackrel{\alpha_{U}}{\rightarrow} \Gamma(G(U), \mathscr{A}) \stackrel{\beta_{U}}{\rightarrow} \Gamma\left(G(U), \mathscr{A}_{2}\right)
$$

for $U \in \mathfrak{T}_{g}$.

$\operatorname{Ad}(\mathrm{b})$. Let $\mathscr{A}$ be a flabby sheaf on $Y$. Since $G(U)$ is open for $U \in \mathfrak{I}_{g}$, if

$$
s \in \Gamma(U, \mathbf{g}(\mathscr{A}))=\Gamma(G(U), \mathscr{A}),
$$

there is a section $s^{\prime}$ in $\Gamma(T, \mathbf{g}(\mathscr{A}))=\Gamma(Y, \mathscr{A})$ with $s^{\prime} \mid G(U)=s$. Hence, $\mathbf{g}(\mathscr{A})$ is flabby.

$\operatorname{Ad}(\mathrm{c})$, For $\mathscr{A} \in \mathscr{S}(Y), \Gamma(Y, \mathscr{A})=\Gamma(T, \mathrm{~g}(\mathscr{A}))$. If $\alpha: \mathscr{A} \rightarrow \mathscr{B}$ is a sheaf homomorphism in $\mathscr{S}(Y)$, the diagram

$$
\begin{array}{ccc}
\Gamma(Y, \mathscr{A}) & = & \Gamma(T, \mathbf{g}(\mathscr{A})) \\
\downarrow \alpha_{Y} & & \downarrow \mathbf{g}(\alpha) \\
\Gamma(Y, \mathscr{B}) & = & \Gamma(T, \mathbf{g}(\mathscr{B}))
\end{array}
$$

commutes since both vertical correspondences are defined by composing sections with $\alpha$. This establishes the natural equivalence in $4.2(\mathrm{c})$. 
For the closed, continuous surjection $f: X \rightarrow Y$ and the determining collection $\left\{G_{t} \mid t \in T\right\}$, there is a corresponding collection of closed sets in $X$, namely

$$
\left\{f^{-1}\left(G_{t}\right) \mid t \in T\right\}
$$

associated with the relation $g f: X \rightarrow T$.

Proposition 4.3. The collection (4.3.1) is determining and the induced topology $\mathfrak{I}_{g f}$ on $T$ coincides with $\mathfrak{I}_{g}$.

Indeed, 2.1(a) follows from the observation that $f$ is continous and onto.

2.1(b) $f^{-1}\left(G_{t_{1}}\right) \cap f^{-1}\left(G_{t_{2}}\right)=f^{-1}\left(G_{t_{1}} \cap G_{t_{2}}\right)=f^{-1}\left(\cup G_{t}\right)=\cup f^{-1}\left(G_{t}\right)$.

2.1(c) For an open neighborhood $V$ of $f^{-1}\left(G_{t}\right)$, let $V_{1}=f\left(V^{\tilde{}}\right)$.

Since $f$ is closed, $V_{1}$ is an open set which evidently includes $G_{t}$. Hence, some open neighborhood of $G_{t}$ of type $W_{1}=\bigcup G_{t}^{\prime}$ is contained in $V_{1}$. Accordingly, $W=f^{-1}\left(W_{1}\right)$ $=\bigcup f^{-1}\left(G_{t}^{\prime}\right)$ is an open saturated neighborhood of $f^{-1}\left(G_{t}\right)$. It is easily verified that $W \subset V$.

Let $B$ be a closed subset of $Y$ with $B^{\sim}$ saturated. Then $f^{-1}(B)^{\tilde{~}}$ is saturated, since

$$
f^{-1}(B)^{\sim}=f^{-1}\left(B^{\tilde{}}\right)=f^{-1}\left(\bigcup G_{t}\right)=\bigcup f^{-1}\left(G_{t}\right) .
$$

Because $f$ is onto, $g(B)=g f\left(f^{-1}(B)\right)$; hence, $\mathfrak{I}_{g} \subset \mathfrak{I}_{g f}$.

On the other hand, suppose $B$ is a closed subset of $X$ with $B^{\sim}$ saturated. Thus $f(B)$ is closed. If $y \in f(B)^{\tilde{y}}$, then $y=f(x)$ for some $x \in B^{\tilde{y}}$. Then, $x \in f^{-1}\left(G_{t}\right) \subset \tilde{B}$ for some $t$, and since $f$ is onto,

$$
y \in G_{t} \subset f(B)^{\tilde{2}} .
$$

Hence, $f(B) \in \Re$ and therefore, $\mathfrak{I}_{g f} \subset \mathfrak{I}_{g}$.

Consequently, $g f$ determines a functor gf: $\subseteq(X) \rightarrow \subseteq(T)$ also satisfying 1.1(a)-(c). With 1.1(d)-(f) in mind, consider an $f$-cohomomorphism $\lambda: \mathscr{A} \rightarrow \mathscr{B}$. For $V$ open in $Y$, there are homomorphisms

$$
\lambda_{V}: \Gamma(V, \mathscr{A}) \rightarrow \Gamma\left(f^{-1}(V), \mathscr{B}\right)
$$

compatible with the restrictions. By replacing $V$ with $G(U), U \in \mathfrak{I}_{g}$, we see that $\lambda$ determines a presheaf homomorphism and, as a consequence, a sheaf homomor$\operatorname{phism} \mathbf{g}(\lambda): \mathbf{g}(\mathscr{A}) \rightarrow \mathbf{g}(\mathscr{B})$.

Diagram (1.1.2) leads to a commutative diagram

$$
\begin{array}{ccc}
\Gamma(V, \mathscr{A}) & \stackrel{\lambda_{V}}{\rightarrow} & \Gamma\left(f^{-1}(V), \mathscr{B}\right) \\
\downarrow \alpha_{V} & & \downarrow \beta_{f^{-1}(V)} \\
\Gamma\left(V, \mathscr{A}_{1}\right) & \stackrel{\lambda_{1 V}}{\rightarrow} & \Gamma\left(f^{-1}(V), \mathscr{B}_{1}\right)
\end{array}
$$

which in turn establishes (1.1.3) when $V=f^{-1}(U)$. Condition 1.1(f) is clear from the definition of $\mathbf{g}(\lambda)$.

We have demonstrated that $\mathbf{g}$, gf is a functor pair of the type considered in $\$ 1$. Condition 2.1(c) asserts that the neighborhoods of the point $t$ are determined by the neighborhoods of the set $G_{t}$. This permits a determination of the stalks of the sheaf 
$\mathbf{g}(\mathscr{A})$ and ultimately those of the derived sheaf $\mathscr{H}(\mathrm{g}, \mathscr{A})$. In the sequel $U_{t}$ denotes a set in $\mathfrak{T}_{g}$ containing $t$.

Proposition 4.4. (a) $L \Gamma\left(G\left(U_{t}\right), \mathscr{A}\right) \cong \Gamma\left(G_{t}, \mathscr{A}\right)$, whence $\mathbf{g}(\mathscr{A})(t)=\Gamma\left(G_{t}, \mathscr{A}\right)$.

(b) $\mathscr{H}^{p}(\mathbf{g}, \mathscr{A})(t) \cong H^{p}\left(G_{t}, \mathscr{A}\right)$.

$\operatorname{Ad}(\mathrm{a})$. The restriction homomorphisms yield homomorphisms

$$
r_{U_{t}}: \Gamma\left(G\left(U_{t}\right), \mathscr{A}\right) \rightarrow \Gamma\left(G_{t}, \mathscr{A}\right),
$$

and the direct limit over the $U_{t}$ yields the homomorphism

$$
r_{t}: L \Gamma\left(G\left(U_{t}\right), \mathscr{A}\right) \rightarrow \Gamma\left(G_{t}, \mathscr{A}\right) .
$$

Suppose $s \in \Gamma\left(G\left(U_{t}\right), \mathscr{A}\right)$ satisfies $s \mid G_{t}=0$. For some neighborhood $V$ of $G_{t}$, $V \subset G\left(U_{t}\right)$ and $s \mid V=0$. By $2.5(\mathrm{~d})$ a neighborhood $U_{t}^{\prime}$ exists with $G\left(U_{t}^{\prime}\right) \subset V$. For $U_{t}^{\prime \prime}=U_{t} \cap U_{t}^{\prime}, s \mid G\left(U_{t}^{\prime \prime}\right)=0$ and therfore the equivalence class of $s$ in $L \Gamma\left(G\left(U_{t}\right), \mathscr{A}\right)$ is 0 . Accordingly, $r_{t}$ is injective.

Let $s \in \Gamma\left(G_{t}, \mathscr{A}\right)$. Since $Y$ is paracompact and $G_{t}$ is closed, $s$ extends to a neighborhood $V$ of $G_{t}$. Again, by $2.5(\mathrm{~d}) s$ extends to a $G\left(U_{t}\right)$, whence $r_{t}$ is surjective.

It should be noted that the left side of (4.4.1) is $\mathrm{g}(\mathscr{A})(t)$.

Ad(b). The relation

$$
U \rightarrow H^{p}(G(U), \mathscr{A})
$$

for $U \in \mathfrak{I}_{g}$ defines a presheaf on $T$.

For $V \subset U$,

$$
i^{*}: H^{p}(G(U), \mathscr{A}) \rightarrow H^{p}(G(V), \mathscr{A}),
$$

where $i: G(V) \stackrel{\subset}{\rightarrow} G(U)$, is the restriction homomorphism for this presheaf. We assert:

(4.4.3) $\mathscr{H}^{p}(\mathrm{~g}, \mathscr{A})$ is the sheaf generated by the presheaf (4.4.2).

Indeed, for the flabby resolution (1.2.1) and $U \in \mathfrak{I}_{g}$,

$$
0 \rightarrow \mathscr{A}\left|G(U) \rightarrow \mathscr{C}^{0}\right| G(U) \rightarrow \mathscr{C}^{1} \mid G(U) \rightarrow \cdots
$$

is a flabby resolution of $\mathscr{A} \mid G(U)$. The corresponding sequence of sections is

$$
\Gamma\left(G(U), \mathscr{C}^{0}\right) \rightarrow \Gamma\left(G(U), \mathscr{C}^{1}\right) \rightarrow \cdots
$$

and $H^{p}(G(U), \mathscr{A})=H^{p}\left(\Gamma\left(G(U), \mathscr{C}^{*}\right)\right)$. The functor taking presheaves to their associated sheaves is exact and therefore commutes with the cohomology functor. Consequently, the sheaf associated with (4.4.2) is the cohomology of the chain complex of sheaves generated by the presheaves in (4.4.5) (namely $U \rightarrow$ $\left.\Gamma\left(G(U), \mathscr{C}^{p}\right)\right)$. But $\Gamma\left(G(U), \mathscr{C}^{p}\right)=\Gamma\left(U, \mathbf{g}\left(\mathscr{C}^{p}\right)\right)$, whence (4.4.5) yields the chain complex

$$
\Gamma\left(U, \mathbf{g}\left(\mathscr{C}^{0}\right)\right) \rightarrow \Gamma\left(U, \mathbf{g}\left(\mathscr{C}^{1}\right)\right) \rightarrow \Gamma\left(U, \mathbf{g}\left(\mathscr{C}^{2}\right)\right) \rightarrow \cdots .
$$

Thus (4.4.3) is a consequence of (1.2.3). Similar results hold for gf according to 4.3.

The paracompactness of $Y$ ensures that

$$
\mathscr{A}\left|G_{t} \rightarrow \mathscr{C}^{0}\right| G_{t} \rightarrow \mathscr{C}^{1} \mid G_{t} \rightarrow \cdots
$$


is a soft resolution of $\mathscr{A} \mid G_{t}$. The combination of 4.4(a), (4.4.3) and the exactness of the direct limit functor leads to

$$
\begin{aligned}
H^{p}\left(G_{t}, \mathscr{A}\right) & =H^{p}\left(\Gamma\left(G_{t}, \mathscr{C}^{*}\right)\right)=H^{p}\left(\underline{L} \Gamma\left(G\left(U_{t}\right), \mathscr{C}^{*}\right)\right) \\
& =\underline{L} H^{p}\left(\Gamma\left(G\left(U_{t}\right), \mathscr{C}^{*}\right)\right)=\underline{L} H^{p}\left(G\left(U_{t}\right), \mathscr{A}\right)=\mathscr{H}^{p}(\mathbf{g}, \mathscr{A})(t) .
\end{aligned}
$$

This verifies 4.4 (b).

For $B \subset Y$, let $f_{B}=f \mid f^{-1}(B): f^{-1}(B) \rightarrow B$. Since $f^{*} \mathscr{A}\left|f^{-1}(B)=f^{*} \mathscr{A}\right| B$, there is an $f_{B}$-cohomorphism

$$
\psi_{B}: \mathscr{A}\left|B \rightarrow f^{*} \mathscr{A}\right| f^{-1}(B)
$$

and a corresponding homomorphism

$$
\psi_{B}^{*}: H^{*}(B, \mathscr{A}) \rightarrow H^{*}\left(f^{-1}(B), f^{*} \mathscr{A}\right) .
$$

(Observe that $\psi_{Y}$ and $\psi_{Y}^{*}$ are the functions $\psi$ and $\psi^{*}$ in (1.5.1) and (1.5.2).)

The inclusion map $i_{B}: B \rightarrow Y$ determines an $i_{B}$-cohomomorphism $\mathscr{A} \rightarrow i_{B}^{*}(\mathscr{A})=$ $\mathscr{A} \mid B$.

(4.5.1) The corresponding homomorphism of sections

$$
\Gamma(U, \mathscr{A}) \rightarrow \Gamma(U \cap B, \mathscr{A} \mid U \cap B)
$$

is merely the restriction homomorphism. Clearly, the following diagram commutes, since $f i_{f^{-1}(B)}=i_{B} f_{B}$ :

$$
\begin{array}{ccc}
H^{*}(Y, \mathscr{A}) & \stackrel{i_{B}^{*}}{\rightarrow} & H^{*}(B, \mathscr{A}) \\
\downarrow \psi^{*} & & \downarrow \psi_{B}^{*} \\
H^{*}\left(X, f^{*} \mathscr{A}\right) & \stackrel{i_{f^{-1}(B)}}{\rightarrow} & H^{*}\left(f^{-1}(B), f^{*} \mathscr{A}\right)
\end{array}
$$

The restriction to $B$ induces a chain map

$$
\begin{array}{cccccc}
\Gamma(Y, \mathscr{A}) & \rightarrow & \Gamma\left(Y, \mathscr{C}^{0}\right) & \rightarrow & \Gamma\left(Y, \mathscr{C}^{1}\right) & \rightarrow \cdots \\
\downarrow r_{Y}^{B} & & \downarrow r_{Y}^{B} & & \downarrow r_{Y}^{B} & \\
\Gamma(B, \mathscr{A}) & \rightarrow & \Gamma\left(B, \mathscr{C}^{0}\right) & \rightarrow & \Gamma\left(B, \mathscr{C}^{1}\right) & \rightarrow \cdots
\end{array}
$$

using the flabby resolution (1.2.1). When $B$ is either open or closed $0 \rightarrow \mathscr{A} \mid B \rightarrow$ $\mathscr{C}^{0}\left|B \rightarrow \mathscr{C}^{1}\right| B \rightarrow \cdots$ is either a flabby or a soft resolution, (4.5.3) determines a homomorphism $r_{B}^{*}: H^{*}(Y, \mathscr{A}) \rightarrow H^{*}(B, \mathscr{A})$. From (4.5.1) follows

$$
i_{B}^{*}=r_{B}^{*} \text {. }
$$

Proposition 4.6. The following diagram commutes:

$$
\begin{array}{ccc}
\mathscr{H}^{q}(\mathbf{g}, \mathscr{A})(t) & \stackrel{\approx}{\rightarrow} & H^{q}\left(G_{t}, \mathscr{A}\right) \\
\downarrow \mathbf{g}\left(\psi^{*}\right)(t) & & \downarrow \psi_{G_{t}}^{*} \\
\mathscr{H}^{q}\left(\mathbf{g f}, f^{*} \mathscr{A}\right)(t) & \stackrel{\sim}{\rightarrow} & H^{q}\left(f^{-1}\left(G_{t}\right), f^{*} \mathscr{A}\right)
\end{array}
$$

(Cf. 4.4 (b) applied to both $\mathrm{g}$ and gf.) 
For $t \in U \in \mathfrak{I}_{g}$ and inclusions $i$ and $j$,

$$
\begin{array}{ccc}
f^{-1}\left(G_{t}\right) & \stackrel{j}{\rightarrow} & f^{-1}(G(U)) \\
\downarrow f_{G_{t}} & & \downarrow f_{G(U)} \\
G_{t} & \stackrel{i}{\rightarrow} & G(U)
\end{array}
$$

commutes. From this information and from (4.5.2) and (4.5.4) we infer that the diagram

$$
\begin{array}{ccc}
H^{q}(G(U), \mathscr{A}) & \stackrel{\left(r_{t}^{U}\right)^{*}}{\rightarrow} & H^{q}\left(G_{t}, \mathscr{A}\right) \\
\downarrow \psi_{G(U)}^{*} & & \downarrow \psi_{G_{t}}^{*} \\
H^{q}\left(f^{-1}(G(U)), f^{*} \mathscr{A}\right) & \stackrel{\left(\hat{r}_{t}^{U}\right)^{*}}{\rightarrow} & H^{q}\left(f^{-1}\left(G_{t}\right), f^{*} \mathscr{A}\right)
\end{array}
$$

commutes. Accordingly:

$$
\begin{array}{ccccc}
\mathscr{H}^{q}(\mathbf{g}, \mathscr{A})(t) & = & L H^{q}\left(G\left(U_{t}\right), \mathscr{A}\right) & \stackrel{\approx}{\rightarrow} & H^{q}\left(G_{t}, \mathscr{A}\right) \\
\downarrow \mathbf{g}(\psi)^{*}(t) & \downarrow L \psi_{G}^{*}\left(U_{t}\right) & & \downarrow \psi_{G_{t}}^{*} \\
\mathscr{H}^{q}\left(\mathbf{g f}, f^{*} \mathscr{A}\right)(t) & = & \underline{L} H^{q}\left(f^{-1}\left(G\left(U_{t}\right)\right), f^{*} \mathscr{A}\right) & \stackrel{\approx}{\rightarrow} & H^{q}\left(f^{-1}\left(G_{t}\right), f^{*} \mathscr{A}\right)
\end{array}
$$

Proposition 4.6 implies the central result:

4.7. For any $t, \psi_{G_{t}}^{*}$ is a monomorphism, epimorphism, or an isomorphism if and only if $\mathrm{g}(\psi)^{*}(t)$ is of the same type.

The difficulty alluded to in the introduction, namely the satisfaction of $S_{2}$, [7], by the presheaf for $\mathbf{g}(A)$ is circumvented by the introduction of $\mathfrak{Z}_{g}$. The crucial property in this regard is $2.5(\mathrm{~b})$, as is evidenced in the course of the demonstration of (4.1.2). Moreover (cf. 2.5(b)), if $U_{1} \cap U_{2}=\varnothing$, then $G\left(U_{1}\right) \cap G\left(U_{2}\right)=\varnothing$. Consequently, when $G_{t_{1}} \cap G_{t_{2}} \neq \varnothing, t_{1}$ and $t_{2}$ cannot be separated by disjoint open sets in $\mathfrak{I}_{g}$, thus $\mathfrak{I}_{g}$ is not Hausdorff whenever the determining collection contains two overlapping sets.

Remark that although 2.1(c) implies

$$
\cap G\left(U_{t}\right)=G_{t}
$$

(4.8.1) cannot substitute for 2.1(c). In particular, (4.8.1) is not sufficient to guarantee 4.4. For instance, in Example 3.5, let $\mathscr{A}$ be the sheaf on $R^{1}$ whose stalks are

$$
\mathscr{A}_{x}= \begin{cases}0 & \text { if } x \notin V \\ J_{2}=\{0,1\} & \text { if } x \in V .\end{cases}
$$

Then $H^{0}(J, \mathscr{A})=\Gamma(J, \mathscr{A})=\oplus_{J} J_{2}$, the direct sum of a denumerable number of copies of $J_{2}$.

For $\varepsilon>0$, let $I_{\varepsilon}$ be the open interval $(-\varepsilon, \varepsilon)$ and for $n \in J$, let $n+I_{\varepsilon}$ be the translation of $I_{\varepsilon}$. For $\varepsilon<1 / 2, J+I_{\varepsilon}$ is the disjoint union of the set $\left\{n+I_{\varepsilon} \mid n \in J\right\}$. 
If $U=\bigcup J+t$ is an open saturated set, then $U=J+U$. Hence, for some $\varepsilon$, $0<\varepsilon<1 / 2$,

$$
J+I_{\varepsilon} \subset U \text {. }
$$

Denote $J+I_{\varepsilon}$ by $W_{\varepsilon}$. For some bound $K, I_{\varepsilon} \subset I_{\varepsilon_{n}}$ if and only if $|n| \leqslant K$. Since $\mathscr{A}$ is 0 in the complement of $V=\bigcup_{n \in J} n+I_{\varepsilon_{n}}$,

$$
\Gamma\left(n+I_{\varepsilon}, \mathscr{A}\right)= \begin{cases}0 & \text { if }|n|>K, \\ J_{2} & \text { if }|n| \leqslant K .\end{cases}
$$

Consequently,

$$
\Gamma\left(W_{\varepsilon}, \mathscr{A}\right)=\bigoplus_{i=1}^{2 K+1} J_{2} .
$$

From $U_{\varepsilon}=g\left(\tilde{W_{\varepsilon}}\right) \tilde{} \in \mathfrak{B}_{g}$ follows $G\left(U_{\varepsilon}\right)=W_{\varepsilon}$. By (4.8.2), $\left\{G\left(U_{\varepsilon}\right) \mid 0<\varepsilon<1 / 2\right\}$ is cofinal in $\left\{G(U) \mid 0 \in U \in \mathfrak{I}_{g}\right\}$ and by (4.8.3)

$$
H^{0}\left(G\left(U_{\varepsilon}\right), \mathscr{A}\right)=\bigoplus_{i=1}^{m} J_{2}
$$

for some finite $m$. Consequently

$$
\mathscr{H}^{0}(\mathbf{g}, \mathscr{A})(0)=\underset{0<\varepsilon<1 / 2}{L} H^{0}\left(G\left(U_{\varepsilon}\right), \mathscr{A}\right)
$$

is the weak direct sum $\oplus_{J}^{W} J_{2}$. Thus

$$
\mathscr{H}^{0}(\mathbf{g}, \mathscr{A})(0) \approx H^{0}\left(G_{0}, \mathscr{A}\right)=H^{0}(J, \mathscr{A}) .
$$

Yet $\cap\left\{G(U) \mid 0 \in U \in \mathfrak{I}_{g}\right\}=G_{0}=J$ because

$$
\bigcap_{0<\varepsilon<1 / 2} G\left(U_{\varepsilon}\right)=\bigcap_{0<\varepsilon<1 / 2} J+I_{\varepsilon}=J .
$$

5. The spectral equivalences. Throughout this section all sets are understood to be groups and all functions are homomorphisms. Moreover, assume that

$$
\left\{\lambda_{r}^{p q}, \psi^{*}\right\}:\left\{E_{r}^{p q}, E^{*}\right\} \rightarrow\left\{\hat{E}_{r}^{p q}, \hat{E}^{*}\right\}
$$

and that (cf. 1.7)

$$
E_{r}^{p q}=\hat{E}_{r}^{p q}=0 \text { for } p<0 \text { or } q<0 .
$$

According to (5.1.2) both homomorphisms

$$
E_{r}^{p-r, q+r-1} \stackrel{d_{r}}{\rightarrow} E_{r}^{p q} \stackrel{d_{r}}{\rightarrow} E_{r}^{p+r, q-r+1}
$$

are trivial for sufficiently large $r$, whence

$$
E_{r}^{p q} \cong E_{\propto}^{p q} \text { for such } r .
$$


Since the homomorphisms $\lambda_{r}^{p q}$ commute with the $d_{r}$ homomorphisms and are compatible with the identifications (1.6.1),

$$
\begin{array}{lll}
E_{r}^{p q} & \stackrel{\equiv}{ } & E_{\infty}^{p q} \\
\downarrow \lambda_{r}^{p q} & & \downarrow \lambda_{\infty}^{p q} \\
\hat{E}_{r}^{p q} & \stackrel{\cong}{\rightarrow} & \hat{E}_{\infty}^{p q}
\end{array}
$$

commutes for large $r$.

Set $C^{n}=\oplus_{t=0}^{n} \Gamma\left(T, \mathscr{B}^{t, n-t}\right)$ with filtration $F_{p} C^{n}=\oplus_{t=p}^{n} \Gamma\left(T, \mathscr{B}^{t, n-t}\right)$, whence $F_{p} C^{n}=0$ for $p>n$. The target $H^{n}\left(C^{*}\right)$ of the spectral sequence $E_{r}^{p q}$ is filtered by

$$
F_{p} H^{n}\left(C^{*}\right)=\operatorname{Im} \alpha^{*}: H^{n}\left(F_{p} C^{*}\right) \rightarrow H^{n}\left(C^{*}\right),
$$

where $\alpha$ is the inclusion map $F_{p} C^{*} \subset C^{*}$. Consequently,

$$
F_{p} E^{n}=F_{p} H^{n}\left(C^{*}\right)=0 \text { for } p>n \text {. }
$$

Similar conclusions are available for $\left\{\hat{E}_{r}^{p q}, \hat{E}^{*}\right\}$.

LEMMA 5.2. Consider the commutative diagram with exact rows:

$$
\begin{array}{lllllllll}
0 & \rightarrow & A & \stackrel{\alpha}{\rightarrow} & B & \stackrel{\beta}{\rightarrow} & C & \rightarrow & 0 \\
& & \downarrow f & & \downarrow g & & \downarrow h & & \\
0 & \rightarrow & A_{1} & \stackrel{\alpha_{1}}{\rightarrow} & B_{1} & \stackrel{\beta_{1}}{\rightarrow} & C_{1} & \rightarrow & 0
\end{array}
$$

5.2(a) If $f$ and $h$ are injective, so is $g$.

5.2(b) If $f$ and $h$ are surjective, so is $g$.

5.2(c) If $f$ and $h$ are isomorphisms, so is $g$.

The verifications are obvious.

The filtration preserving homomorphism $\psi^{n}: E^{n} \rightarrow \hat{E}^{n}$ of 1.6 induces homomorphisms $\psi_{p}^{n}: F_{p} E^{n} \rightarrow F_{p} \hat{E}^{n}$ and

$$
\tilde{\psi}_{p}^{n}: F_{p} E^{n} / F_{p+1} E^{n} \rightarrow F_{p} \hat{E}^{n} / F_{p+1} \hat{E}^{n} .
$$

Corollary 5.3. Assume the filtrations on $E^{n}$ and $\hat{E}^{n}$ satisfy (5.1.5). For each $n$, if $\tilde{\psi}_{p}^{n}$ is (a) injective, (b) surjective, (c) bijective for every $p$, then $\psi^{n}$ is (a) injective, (b) surjective, (c) bijective .

Consider the following commutative diagram with exact rows:

$$
\begin{aligned}
& 0 \rightarrow F_{p+1} E^{n} \rightarrow F_{p} E^{n} \rightarrow \frac{F_{p} E^{n}}{F_{p+1} E^{n}} \rightarrow 0
\end{aligned}
$$

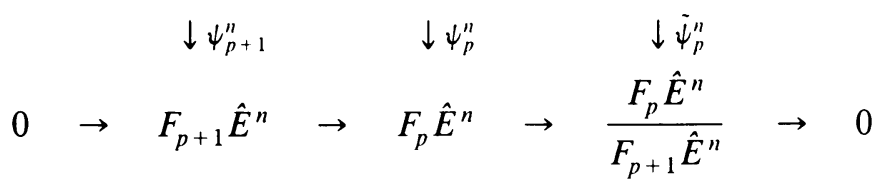

For (a) assume each $\tilde{\psi}_{p}^{n}$ is one-to-one. By (5.1.5), $F_{n+1} E^{n}=F_{n+1} \hat{E}^{n}=0$, hence $\psi_{n+1}^{n}$ is injective too. 
An application of 5.2(a) shows $\psi_{n}^{n}$ is injective. An induction argument using 5.2(a) again establishes that $\psi_{p}^{n}$ is injective for all $p$. Then from

$$
E^{n}=\bigcup_{p} F_{p} E^{n}, \quad \hat{E}^{n}=\bigcup_{p} F_{p} \hat{E}^{n}
$$

and

$$
\psi^{n}=\bigcup_{p} \psi_{p}^{n}: \bigcup_{p} F_{p} E^{n} \rightarrow \bigcup_{p} F_{p} \hat{E}^{n}
$$

we infer that $\psi^{n}$ is injective also.

The same line of reasoning starting with 5.2(b) yields 5.3(b). Assertion 5.3(c) follows directly from 5.3(a) and (b).

LEMMA 5.4. For the commutative diagram

$$
\begin{array}{lllll}
A & \stackrel{d}{\rightarrow} & B & \stackrel{d^{\prime}}{\rightarrow} & C \\
\downarrow \alpha & & \downarrow \beta & & \downarrow \gamma \\
A_{1} & \stackrel{d_{1}}{\rightarrow} & B_{1} & \stackrel{d_{1}^{\prime}}{\rightarrow} & C_{1}
\end{array}
$$

where $d^{\prime} d=d_{1}^{\prime} d_{1}=0$, define $H=\operatorname{ker} d^{\prime} / \operatorname{Im} d, H_{1}=\operatorname{ker} d_{1}^{\prime} / \operatorname{Im} d_{1}$ and let $\beta^{*}$ : $H \rightarrow H_{1}$ be the induced homomorphism. Then:

5.4(a) $\beta^{*}$ is onto whenever $\beta$ is onto and $\gamma$ is one-to-one.

5.4(b) $\beta^{*}$ is one-to-one whenever $\alpha$ is onto and $\beta$ is one-to-one, and

5.4(c) $\beta^{*}$ is an isomorphism whenever $\beta$ is an isomorphism, $\gamma$ is one-to-one and $\alpha$ is onto.

$\operatorname{Ad}(a)$. If $\left\langle b_{1}\right\rangle \in H_{1}, b_{1}=\beta(b)$ for some $b$ since $\beta$ is onto. Thus

$$
\gamma d^{\prime}(b)=d_{1}^{\prime} \beta(b)=d_{1}^{\prime}\left(b_{1}\right)=0,
$$

and so, since $\gamma$ is one-to-one, $d^{\prime}(b)=0$. Therefore, $\langle b\rangle \in H$ and $\beta^{*}(\langle b\rangle)=$ $\langle\beta(b)\rangle=\left\langle b_{1}\right\rangle$, i.e. $\beta^{*}$ is onto.

$\operatorname{Ad}(\mathrm{b})$. If $\langle b\rangle \in H$ with $\beta^{*}\langle b\rangle=0$, then $\beta(b) \in \operatorname{Im} d_{1}$, and so $\beta(b)=d_{1}\left(a_{1}\right)$ for $a_{1} \in A_{1}$. Since $\alpha$ is onto, $\alpha(a)=a_{1}$ for $a \in A$ and

$$
\beta d(a)=d_{1} \alpha(a)=d_{1}\left(a_{1}\right)=\beta(b) .
$$

By the injectivity of $\beta, d(a)=b$ and thus $\langle b\rangle=0$ in $H$. In sum, $\beta^{*}$ is one-to-one.

Clearly, 5.4(c) is a direct consequence of (a) and (b).

The underlying plan for the proof of the next theorem is patterned on that for a somewhat analogous situation in [2]. However, the introduction of diagram (5.5.1) allows a more direct presentation.

Let $\psi^{*}: E^{*} \rightarrow \hat{E}^{*}$ be a homomorphism of graded groups. Fix integers $n<N$. If $\psi^{p}$ is an isomorphism for $n<p<N, \psi$ is an $(n, N)$ homomorphism. If, in addition, $\psi^{n}$ is surjective or $\psi^{N}$ is injective, then $\psi$ is an $[n, N)$ or $(n, N]$ homomorphism respectively. The symbol $[n, N]$ connotes satisfaction of both conditions. This terminology is extended to a homomorphism $\lambda_{r}^{p q}: E_{r}^{p q} \rightarrow \hat{E}_{r}^{p q}$ where the conditions of $\lambda_{r}^{p q}$ are imposed under the restriction $n<p+q<N$ or $p+q=n$ or $p+q=N$. 
THEOREM 5.5. Let $\left\{\lambda_{r}^{p q}, \psi^{*}\right\},\left\{E_{r}^{p q}, E^{*}\right\} \rightarrow\left\{\hat{E}_{r}^{p q}, \hat{E}^{*}\right\}$ for which $E_{r}^{p q}=\hat{E}_{r}^{p q}=0$ if $p<0$ or $q<0$. Assume for fixed integers $n<N$ that:

5.5(a) $\lambda_{2}^{p q}$ is an isomorphism whenever $n<p+q<N$,

5.5(b) $\lambda_{2}^{p q}$ is surjective whenever $p+q=n$, and

5.5(c) $\lambda_{2}^{p q}$ is injective for $p+q=N$ and $q \leqslant N-2$.

Then $\psi^{*}$ is an $[n, N)$ homomorphism.

Furthermore, if

5.5(d) $\lambda_{2}^{p q}$ is injective for $p+q=N$, then $\psi^{*}$ is an $[n, N]$ homomorphism.

The demonstration consists of evident application of Lemma 5.4 to

$$
\begin{aligned}
& E_{r}^{p-r . q+r-1} \rightarrow E_{r}^{p q} \rightarrow E_{r}^{p+r, q-r+1} \\
& \downarrow \lambda_{r}^{p \cdot q+r \cdot 1} \quad \downarrow \lambda_{r}^{p q} \quad \downarrow \lambda_{r}^{p+r \cdot q} \cdot r+1 \\
& \hat{E}_{r}^{p-r . q+r-1} \quad \rightarrow \quad \hat{E}_{r}^{p q} \quad \rightarrow \quad \hat{E}_{r}^{p+r \cdot q-r+1}
\end{aligned}
$$

for $2 \leqslant r . \lambda_{r}^{p-r \cdot q+r-1}, \lambda_{r}^{p q}$ and $\lambda_{r}^{p+r, q-r+1}$ are referred to as the first, second and third $\lambda_{r}$ respectively.

An induction argument will show that assumptions (a)-(c), or (a), (b) and (d), imply their counterparts for $\lambda_{r}^{p q}$ where $r \geqslant 2$. Accordingly, we stipulate that the conditions are satisfied for $r$ by $\left\{\lambda_{r}^{p q}\right\}$ and then show they are satisfied by $\left\{\lambda_{r+1}^{p q}\right\}$. For $n<p+q<N$,

$$
n<(p+r)+(q-r+1) \leqslant N \text { and } n \leqslant(p-r)+(q+r-1)<N,
$$

whence the first $\lambda_{r}$ in (5.5.1) is surjective and the second is bijective. Therefore, $\left(\lambda_{r}^{p q}\right)^{*}=\lambda_{r+1}^{p q}$ is injective by 5.4(b). If $p+q<N-1$, the third $\lambda_{r}$ is bijective and so by 5.4(a) $\lambda_{r+1}^{p q}$ is surjective. If $p+q=N-1$ and $q-r+1 \leqslant N-2$, the third $\lambda_{r}$ is injective and again $\lambda_{r+1}^{p q}$ is surjective. Finally, if $p+q=N-1$ and $q-r+1$ $>N-2$, then $p<0$ and $\lambda_{r+1}^{p q}$ is surjective because $E_{r}^{p q}=\hat{E}_{r}^{p q}=0$. Accordingly, $\lambda_{r+1}^{p q}$ is an isomorphism whenever $n<p+q<N$.

For $p+q=n, \lambda_{r}^{p q}$ is surjective and $\lambda_{r}^{p+r, q-r+1}$ is injective. (Note, when $p+q+$ $1=N$, either $q-r+1 \leqslant N-2$ or $p<0$.) Accordingly, $\lambda_{r+1}^{p q}$ is surjective.

If $p+q=N$, then $n \leqslant p+q-1<N$. Thus $\lambda_{r}^{p-r \cdot q+r-1}$ is surjective and $\lambda_{r}^{p q}$ is injective (provided further that $q \leqslant N-2$ for condition (c)), whence $\lambda_{r+1}^{p q}$ is injective. This completes the inductive step.

Next, according to (5.1.4), $\lambda_{\propto}^{p q}$ satisfies 5.5(a), (b) and (c) or 5.5(a), (b) and (d) whenever these conditions are satisfied by $\lambda_{r}^{p q}, r \geqslant 2$. Hence, bearing in mind (1.6.2), $\tilde{\psi}_{p}^{p q}$ is an isomorphism for $n<p+q<N$, a surjection for $p+q=n$ and, under assumption 5.5(d), an injection for $p+q=N$. An application of Corollary 5.3 completes the demonstration.

THEOREM 5.6. Under the basic assumptions of Theorem 5.5, if

5.6(a) $\lambda_{2}^{p q}$ is surjective for $n \leqslant p+q<N+p$ and $0<p$,

5.6(b) $\lambda_{2}^{p q}$ is injective for $n<p+q<N+p$ and $1<p$, and

$5.6(\mathrm{c}) \psi^{q}$ is an $[n, N]$ homomorphism, then

5.6(d) $\lambda_{2}^{0 q}$ is an $[n, N]$ homomorphism and

5.6(e) $\lambda_{2}^{1 q}$ is an $[n-1, N)$ homomorphism. 
The technique of proof is probably aptly described as "up the ladder and down again." The information about $\lambda_{2}^{p q}$ embodied in 5.6(a) and (b) is carried up to the $\lambda_{\infty}^{p q}$ level in Step 1. In Step 2 this is combined with 5.6(c). These results are then brought back to the $r=2$ level to establish 5.6(d) and (e) in Step 3.

Step 1. Using induction on $r$ we show that

$$
\begin{aligned}
& \lambda_{r}^{p q} \text { is surjective if } n \leqslant p+q<N+p \text { and } p>0, \\
& \lambda_{r}^{p q} \text { is injective if } n<p+q<N+p-r+2 \text { and } p \geqslant r .
\end{aligned}
$$

Note (5.6.1) is valid by hypothesis when $r=2$. Assume (5.6.1) holds for $r$. Proceed from diagram (5.5.1). For $n \leqslant p+q<N+p$ and $0<p, \lambda_{r}^{p q}$ is surjective and $\lambda_{r}^{p+r, q-r+1}$ is injective, thus $\lambda_{r+1}^{p q}$ is surjective by 5.4(a). If

$$
\text { - } n<p+q<N+p-(r+1)+2=N+p-r+1
$$

and $r+1 \leqslant p$, then

$$
n<p+q<N+p-r+2, \quad r \leqslant p,
$$

and so $\lambda_{r}^{p q}$ is injective. Furthermore, $n \leqslant p+q-1<N+(p-r)$ and $0<p-r$, so $\lambda_{r}^{p-r, q+r-1}$ is surjective. Consequently, $\lambda_{r+1}^{p q}$ is injective.

Step 2. Next we show that

$\lambda_{\infty}^{p q}$ is an epimorphism if $n \leqslant p+q<N$ or if $p+q=N$ and $p>0$,

$\lambda_{\infty}^{p q}$ is a monomorphism if $n<p+q \leqslant N$.

In the notation of 5.3 ,

$$
F_{p} E^{k} / F_{p+1} E^{k}=E_{\infty}^{p, k-p}=0 \text { for } p<0,
$$

and so $F_{0} E^{k}=E^{k}$; similarly $F_{0} \hat{E}^{k}=\hat{E}^{k}$. Consider the commutative diagram:

$$
\begin{aligned}
& E^{q} \rightarrow E^{q} / F_{1} E^{q} \cong E_{\infty}^{0 q} \\
& \downarrow \psi^{q} \quad \downarrow \quad \downarrow \lambda_{\infty}^{0 q} \\
& \hat{E}^{q} \quad \rightarrow \quad \hat{E}^{q} / F_{1} \hat{E}^{q} \cong \hat{E}_{\infty}^{0 q}
\end{aligned}
$$

For $n \leqslant q<N, \psi^{q}$ is surjective by 5.6(c) and, therefore, so is $\lambda_{\infty}^{0 q}$.

For $p>0,(5.6 .2)$ follows from Step 1 and (5.1.4).

Now, suppose $n<p+q \leqslant N$. Since $\psi^{p+q}$ is injective,

$$
\psi_{p}^{p+q}: F_{p} E^{p+q} \rightarrow F_{p} \hat{E}^{p+q} \text { is injective. }
$$

There is a commutative diagram with exact rows:

$$
\begin{array}{ccccccccc}
0 & \rightarrow & F_{p+1} E^{p+q} & \stackrel{\alpha}{\rightarrow} & F_{p} E^{p+q} & \stackrel{\beta}{\rightarrow} & E_{\infty}^{p q} & \rightarrow & 0 \\
& & \downarrow \psi_{p+1}^{p+q} & & \downarrow \psi_{p}^{p+q} & & \downarrow \lambda_{\infty}^{p q} & & \\
0 & \rightarrow & F_{p+1} \hat{E}^{p+q} & \stackrel{\alpha^{\prime}}{\rightarrow} & F_{p} \hat{E}^{p+q} & \stackrel{\beta^{\prime}}{\rightarrow} & \hat{E}_{\infty}^{p q} & \rightarrow & 0
\end{array}
$$

By (5.6.1) $\lambda_{\infty}^{p q}$ is surjective for $n<p+q \leqslant N$ and $p>0$. For $k=p+q$ fixed, with $n<k \leqslant N, \psi_{p+1}^{k}$ is surjective for $p \geqslant k$ by (5.1.5). Thus in view of 5.2(b) $\psi_{p}^{k}$ is surjective. Induction, reducing $p$, establishes that $\psi_{p}^{p+q}$ is a surjection for $p>0$ and 
$n<p+q \leqslant N$. Return to diagram (5.6.5) for $n<p+q \leqslant N$ and $p \geqslant 0$ to conclude that $\psi_{p+1}^{p+q}$ is surjective and, by (5.6.4), that $\psi_{p}^{p+q}$ is injective. Diagram chasing verifies that $\lambda_{\infty}^{p q}$ is injective. This disposes of (5.6.3).

Step 3. For $r \geqslant 2$ we show that

$\lambda_{r}^{p q}$ is an epimorphism if $n \leqslant p+q<N$

or $p+q=N$ and $p>0$, and is a monomorphism if $n<p+q \leqslant N$.

In particular, this establishes 5.6(d) and (e) when $p=0$ and $p=1$ respectively.

In view of (5.6.2) and (5.1.4), condition (5.6.6) is valid for sufficiently large $r$. The proof is completed by downward induction on $r$ for (5.6.6) when $r \geqslant 2$. Thus assume:

(a) $\lambda_{r+1}^{p q}$ is surjective for $n \leqslant p+q<N$ or $p+q=N$ and $p>0$,

(b) $\lambda_{r+1}^{p q}$ is injective for $n<p+q \leqslant N$,

(c) $\lambda_{r}^{p q}$ is surjective for $n \leqslant p+q<N+p$ and $p>0$, and

(d) $\lambda_{r}^{p q}$ is injective for $n<p+q<N+p-r+2$ and $p \geqslant r$,

where (c) and (d) were established in Step 1. To show (a) for $\lambda_{r}^{p q}$ it is sufficient, by reason of (c), to take $p=0$ and $n \leqslant q<N$. In the commutative diagram

$$
\begin{aligned}
& 0 \rightarrow E_{r}^{0 q} \stackrel{d_{r}}{\rightarrow} E_{r}^{r, q-r+1} \stackrel{d_{r}^{\prime}}{\rightarrow} E_{r}^{2 r, q-2 r+2} \\
& \downarrow \alpha \quad \downarrow \beta \quad \downarrow \gamma \\
& 0 \rightarrow \hat{E}_{r}^{0 q} \stackrel{\hat{d}_{r}}{\rightarrow} \hat{E}_{r}^{r, q-r+1} \stackrel{\hat{d}_{r}^{\prime}}{\rightarrow} \quad \hat{E}_{r}^{2 r, q-2 r+2}
\end{aligned}
$$

where $\alpha=\lambda_{r}^{0 q}, \alpha^{*}=\lambda_{r+1}^{0 q}, \beta=\lambda_{r}^{r q-r+1}, \beta^{*}=\lambda_{r+1}^{r, q-r+1}$ and $\gamma=\lambda_{r}^{2 r, q-2 r+2}$,

(e) $\alpha^{*}$ is surjective by (a),

(f) $\beta$ is surjective by (c), since $n<q+1<N+1<N+r$ and $r>0$,

(g) $\beta^{*}$ is injective by (b), and

(h) $\gamma$ is injective by (d), since

$$
n<q+2<N+2<N+(2 r)-r+2 \text { and } 2 r>r .
$$

We show that $\alpha$ is an epimorphism. For $x \in \hat{E}_{r}^{0 q}, \hat{d}_{r}(x)=\beta(y)$ by (f). Since

$$
\gamma d_{r}^{\prime}(y)=\hat{d}_{r}^{\prime} \beta(y)=\hat{d}_{r}^{\prime} \hat{d}_{r}(x)=0,
$$

$d_{r}^{\prime}(y)=0$ by (h). Consequently, $\langle y\rangle \in H\left(E_{r}^{r, q-r+1}\right)$ and $\beta^{*}\langle y\rangle=\left\langle\hat{d}_{r}(x)\right\rangle=0$. Therefore, by $(\mathrm{g}),\langle y\rangle=0$ and so $y=d_{r}(z)$ for some $z \in E_{r}^{0 q}$. Now

$$
\hat{d}_{r}(x-\alpha(z))=\hat{d}_{r}(x)-\hat{d}_{r} \alpha(z)=\beta(y)-\beta d_{r}(z)=0 .
$$

Hence

$$
x-\alpha(z) \in \operatorname{ker}\left(\hat{d}_{r}\right)=H\left(\hat{E}_{r}^{0 q}\right)
$$

and so $x-\alpha(z)=\alpha(w)$ by (e), where $w \in H\left(E_{r}^{0 q}\right)=\operatorname{ker}\left(d_{r}\right)$. Consequently, $x=$ $\alpha(z+w)$; this is to say $\alpha$ is onto. 
To establish (b) for $\lambda_{r}^{p q}$, take $n<p+q \leqslant N$. If $p \geqslant r, n<p+q<N+1<N$ $+p-r+2$ and (d) implies $\lambda_{r}^{p q}$ is one-to-one. For $p<r$, consider the commutative diagram

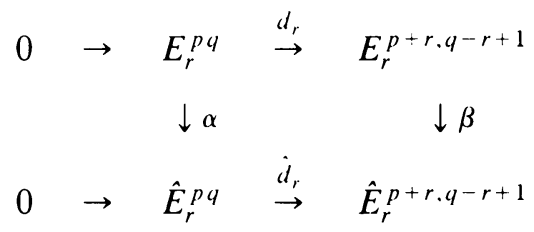

where $\alpha=\lambda_{r}^{p q}, \alpha^{*}=\lambda_{r+1}^{p q}$ and $\beta=\lambda_{r}^{p+r . q-r+1}$ and $E_{r}^{p-r . q+r-1}=\hat{E}_{r}^{p-r . q+r-1}=0$, since $p<r$. By (b) and (d) we know

(i) $\alpha^{*}$ is a monomorphism and

(j) $\beta$ is a monomorphism,

since $n<p+q+1 \leqslant N+1<N+(p+r)-r+2$ and $p+r \geqslant r$. (We assume $p \geqslant 0$ noting $\alpha$ is clearly one-to-one if $p<0$.) Choose $x \in E_{r}^{p q}$ so that $\alpha(x)=0$. Then $d_{r}(x)=0$ by (j) because $\beta d_{r}(x)=\hat{d}_{r} \alpha(x)=0$. Hence, $x \in \operatorname{Ker}\left(d_{r}\right)=H\left(E_{r}^{p q}\right)$ satisfies $\alpha^{*}\langle x\rangle=0$ and so by (i), $x=0$. Consequently, $\alpha$ is one-to-one. This completes Step 3 and the proof of the theorem.

6. Direct and inverse Vietoris-Begle type theorems. We introduce some convenient terminology. A not necessarily proper subset $B$ of $Y$ is a $q$-nonsingular set (with respect to $f$ ) provided

$$
\psi_{B}^{*}: H^{q}(B, \mathscr{A}) \rightarrow H^{q}\left(f^{-1}(B), f^{*} \mathscr{A}\right)
$$

is an isomorphism. Otherwise, $B$ is a $q$-singular set. If $n<N$ and $B$ is a $q$-nonsingular set for $n<q<N$, then $B$ is an $(n, N)$ nonsingular set. Hence, $B$ is an $(n, N)$ singular set whenever $B$ is $q$-singular for some $q, n<q<N$. If $B$ is an $(n, m)$ nonsingular set, and if, in addition

(1) $H^{n}(B, \mathscr{A}) \rightarrow H^{n}\left(f^{-1}(B), f^{*} \mathscr{A}\right)$ is onto, or

(2) $H^{N}(B, \mathscr{A}) \rightarrow H^{N}\left(f^{-1}(B), f^{*} \mathscr{A}\right)$ is one-to-one, or

(3) both (1) and (2) are valid,

then $B$ is (1) $[n, N)$ nonsingular, (2) ( $n, N]$ nonsingular, or (3) $[n, N]$ nonsingular, respectively. This terminology may be extended to points in $T$. Thus, $t$ is $(n, N)$ nonsingular provided $B=G_{t}$ is $(n, N)$ nonsingular.

Definition 6.1. The relative cohomological dimension of the subset $M$ of $T$ is $\operatorname{dim}_{T}(M)=\min \left\{n \geqslant 0 \mid H^{K}(T, \mathscr{A})=0\right.$ for every $K>n$ and every sheaf $\mathscr{A}$ concentrated on $M\}$, if $M \neq \varnothing$, and $\operatorname{dim}_{T}(\phi)=-\infty$.

We borrow Lemma 2 of [2], whose proof depends only on the dimension property used in 6.1, and state it as

Proposition 6.2. Let $\mathscr{A}_{1}$ and $\mathscr{A}_{2}$ be sheaves on $T$ topologized by $\mathfrak{I}_{g}$ and $\alpha$ : $\mathscr{A}_{1} \rightarrow \mathscr{A}_{2}$ be a sheaf homomorphism. If the kernel and cokernel of $\alpha$ are concentrated on sets of relative cohomological dimension $<n$, then the induced homomorphism

$$
\alpha^{*}: H^{p}\left(T, \mathscr{A}_{1}\right) \rightarrow H^{p}\left(T, \mathscr{A}_{2}\right)
$$

is an isomorphism for $p>n$ and an epimorphism for $p=n$. Furthermore, if $\alpha$ is surjective, then $\alpha^{*}$ is an isomorphism for $n=p$. 
For $q \geqslant 0$ define

$$
M_{q}=\left\{t \mid \psi_{G_{t}}^{*}: H^{q}\left(G_{t}, \mathscr{A}\right) \rightarrow H^{q}\left(f^{-1}\left(G_{t}\right), f^{*} \mathscr{A}\right) \text { is not bijective }\right\},
$$

the set of $q$-singular ponts in $T$, and $d_{q}=\operatorname{dim}_{T} M_{q}$.

THEOREM 6.3. If, for $n<N$,

$$
1+d_{q}+q \leqslant n \text { for } 0 \leqslant q<N,
$$

then $\psi^{*}: H^{*}(Y, \mathscr{A}) \rightarrow H^{*}\left(X, f^{*} \mathscr{A}\right)$ is an $[n, N)$ homomorphism.

Furthermore, if

$$
\psi_{G_{t}}^{*}: H^{N}\left(G_{t}, \mathscr{A}\right) \rightarrow H^{N}\left(f^{-1}\left(G_{t}\right), f^{*} \mathscr{A}\right)
$$

is injective for every $t$, then $\psi^{*}$ is an $[n, N]$ homomorphism.

It may be noted that $(6.3)(\mathrm{a})$ is equivalent to

$(6.3 .1)\left(\right.$ a) $\psi_{G_{t}}^{*}$ is an $(n-1, N)$ homomorphism for every $t$, and (b) $d_{q} \leqslant n-q-1$ for $0 \leqslant q \leqslant n-1$.

Hence, to insure that $\psi^{*}$ is an $[n, N)$ homomorphism, it is sufficient that every $\psi_{G_{t}}^{*}$ be an isomorphism in the $n \leqslant q<N$ range and that the dimension of each set of $q$-singular values in $T$ be sufficiently small in the $0 \leqslant q \leqslant n-1$ range.

The first part of 6.3 is proved by an application of 5.5(a)-(c). According to 4.7,

$$
g(\psi)^{*}(t): \mathscr{H}^{q}(\mathbf{g}, \mathscr{A})(t) \rightarrow \mathscr{H}^{q}\left(\mathbf{g f}, f^{*} \mathscr{A}\right)(t)
$$

is an isomorphism if and only if $\psi_{G_{t}}^{*}$ is one. Clearly, then the kernel and cokernel of $g(\psi)^{*}$ are concentrated on $M_{q}$. Consequently, by Proposition 6.2

$$
\lambda_{2}^{p q}: H^{p}\left(T, \mathscr{H}^{q}(\mathbf{g}, \mathscr{A})\right) \rightarrow H^{p}\left(T, \mathscr{H}^{q}\left(\mathbf{g f}, f^{*} \mathscr{A}\right)\right)
$$

is an isomorphism for $p>d_{q}+1$ and an epimorphism for $\dot{p}=d_{q}+1$.

Let $n<p+q<N$. If $q \geqslant N, p<0$ and so $\lambda_{2}^{p q}$ is an isomorphism, and if $q \leqslant N-1, p>n-q \geqslant 1+d_{q}$ by 6.3(a); thus $\lambda_{2}^{p q}$ is an isomorphism by (6.3.3). If $p+q=N$ and $q \leqslant N-1$,

$$
d_{q}+1 \leqslant n-q=p-(N-n)<p
$$

and therefore, $\lambda_{2}^{p q}$ is an isomorphism again. For $p+q=n$; if $q>n$, then $P<0$, and if $q \leqslant n$, then $d_{q}+1 \leqslant n-q=p$. In either case $\lambda_{2}^{p q}$ is an epimorphism. Consequently, conditions 5.5(a)-(c) are established and thus $\psi^{*}$ is an $[n, N)$ homomorphism.

The other assertion of the theorem is established by showing that (6.3)(b) implies 5.5(d). Indeed, for $p+q=N, \lambda_{2}^{p q}$ is an isomorphism if either $q \leqslant N-1$ (as above) or $q>N$ (since $p<0$ ). The case $q=N$ and $p=0$ still remains. Since (6.3)(b) implies that (6.3.2) is injective for $q=N$,

$$
\begin{aligned}
0 & \rightarrow \Gamma\left(T, \mathscr{H}^{N}(\mathbf{g}, \mathscr{A})\right)=H^{0}\left(T, \mathscr{H}^{N}(\mathbf{g}, \mathscr{A})\right) \\
& \rightarrow \Gamma\left(T, \mathscr{H}^{N}\left(\mathbf{g}, f^{*} \mathscr{A}\right)\right)=H^{0}\left(T, \mathscr{H}^{N}\left(\mathbf{g}, f^{*} \mathscr{A}\right)\right)
\end{aligned}
$$

is exact and therefore $\lambda_{2}^{0 N}: E_{2}^{0 N} \rightarrow \hat{E}_{2}^{0 N}$ is one-to-one. This completes the proof of the theorem. 
The following corollary to Theorem 6.3 ensues on taking $n=-1$.

COROLlary 6.4. If

$$
\psi_{G_{t}}^{*}: H^{q}\left(G_{t}, \mathscr{A}\right) \rightarrow H^{q}\left(f^{-1}\left(G_{t}\right), f^{*} \mathscr{A}\right)
$$

is an isomorphism for $0 \leqslant q<N$ and every $t$, then $\psi^{*}: H^{q}(Y, \mathscr{A}) \rightarrow H^{q}\left(X, f^{*} \mathscr{A}\right)$ is an isomorphism for $0 \leqslant q \leqslant N-1$. Furthermore, if (6.4.1) is one-to-one for $q=N$ and every $t$, then $\psi^{*}$ is a monomorphism for $q^{\circ}=N$.

An inference from Example 3.1, for $g$ a continuous, closed surjection, is that Theorem 6.3 reduces to [2, Theorem 3]. (Superficially, it may appear that 6.3 is stronger in this case also since [2] carries the added assumption that $T$ is paracompact. However, the fact that $Y$ is paracompact, together with the conditions placed upon $g$ imply that $T$ is paracompact too [9].)

In Theorem 6.3 for each $N$, the value of $n$ may be computed from

$$
n=\max \left\{1+d_{q}+q \mid 0 \leqslant q<N\right\}
$$

and the theorem applies whenever $n<N$.

In the Vietoris-Begle theorem the points of $Y$ are nonsingular. While in our generalized variations the sets $g^{-1}(t)$ are nonsingular, though they need not be free of singular points. This is evidenced in the following example for constant sheaves and $A$ an abelian group.

EXAMPLE 6.5. Let $C S^{2}$ denote the cone on $S^{2}, S^{2} \times[1 / 2,1]$ with $S^{2} \times 1$ identified to a point, and let $\Sigma S^{1}$ denote the unreduced suspension of $S^{1}$ formed by collapsing the roof and floor of $S^{1} \times[0,1]$ to distinct points. A point in $S^{2} \times 1 / 2 \subset$ $C S^{2}$ and a point in $S^{1} \times 1 / 2 \subset \sum S^{1}$, are identified to give the wedge product $X=C S^{2} \vee \sum S^{1}$. Here, and through (6.5.4), $Y=[0,1]$. The projection $f: X \rightarrow Y$, defined by $f(x, t)=t$, is a continuous, closed surjection.

Since $X$ contracts to $\sum S^{1} \approx S^{2}$,

$$
H^{0}(X, A)=H^{2}(X, A)=H^{0}(Y, A)=A
$$

and the remaining cohomology groups are trivial. Thus $\psi^{*}: H^{*}(Y, A) \rightarrow H^{*}(X, A)$ is a $[3, N)$ homomorphism (in fact, a $(2, N)$ homomorphism).

(6.5.1). Let $T=Y$ and let $g$ be the identity map, then $G_{t}=\{t\}$ and $\mathfrak{I}_{g}$ is the usual topology on $[0,1]$. For this case

$$
M_{q}=\left\{t \mid H^{q}\left(f^{-1}(t), \mathscr{A}\right) \neq 0\right\} \quad \text { for } 0<q
$$

and $M_{0}=\left\{t \mid f^{-1}(t)\right.$ is not connected $\}$. Since $f^{-1}(t)=S^{1}$ if $0<t<1 / 2, f^{-1}(t)=$ $S^{1} \cup S^{2}$ if $1 / 2<t<1, f^{-1}(1 / 2)=S^{1} \vee S^{2}$ and $f^{-1}(0$ or 1$)=$ point,

$$
M_{0}=(1 / 2,1), \quad M_{1}=(0,1) \quad \text { and } \quad M_{2}=[1 / 2,1) .
$$

Consequently, $d_{0}=d_{1}=d_{2}=1$ and $d_{q}=-\infty$ for $q>2$. Hence, for $N \geqslant 3$

$$
n=\max _{0 \leqslant q<N-1} 1+d_{q}+q=4
$$

Accordingly, Theorem 6.3 is not optimal for this relation.

(6.5.2). Next let $T=[0,1 / 4], G_{t}=\{t\}$ for $0 \leqslant t<1 / 4$ and $G_{1 / 4}=[1 / 4,1]$. The collection $\left\{G_{t} \mid t \in T\right\}$ is determining and $\mathfrak{I}_{g}$ is the usual topology on $T$. The set 
$f^{-1}\left(G_{1 / 4}\right)$ is contractible and $f^{-1}\left(G_{t}\right)=S^{1}$ for $0<t<1 / 4$. Thus, $M_{0}=\varnothing, M_{1}=$ $(0,1 / 4)$ and $M_{q}=\varnothing$ for $q \geqslant 2$. Hence, $d_{1}=1$ and $n=3$. In this case, then, Theorem 6.3 yields the expected results.

(6.5.3). Let $T=\{1,2,3\}, G_{1}=[0,1 / 4], G_{2}=\{1 / 4\}$ and $G_{3}=[1 / 4,1]$. The collection $\left\{G_{1}, G_{2}, G_{3}\right\}$ satisfies $2.1(\mathrm{a})-(\mathrm{c})$ but not $2.1(\mathrm{~d})$. These sets result from the closed relation in $Y \times Y$,

$$
g(t)= \begin{cases}0 & \text { if } 0 \leqslant t<1 / 4 \\ Y & \text { if } t=1 / 4 \\ 1 & \text { if } 1 / 4<t \leqslant 1\end{cases}
$$

Since $f^{-1}\left(G_{1}\right)$ and $f^{-1}\left(G_{3}\right)$ are contractible and $f^{-1}\left(G_{2}\right)=S^{1}, M_{1}=\{2\}$ and $M_{q}=\varnothing$ for $q \neq 1$. The only open saturated set is $Y$, thus $\mathfrak{I}_{g}$ is the indiscrete topology on $T$. Consequently, every sheaf on $T$ is flabby, and so every subset has relative cohomological dimension 0 . This implies $d_{1}=0$ and $d_{q}=-\infty$ for $q \neq 1$, whence $n=2$. In this case the results of 6.3 are too good, in fact false. Thus, Theorem 6.3 is invalid for sets satisfying 2.1(a)-(c) only. These restrictions meet the conditions in [5], but not the additional ones of [1, Theorem 9.1], and point to the necessity of some further assumptions like those in the present work or in [1] to extend results of the type in [5 or 6] to the $[n, N]$ range, even for constant sheaves.

(6.5.4). For $T=[0,1 / 4], G_{t}=[t, 1]$ for $0<t \leqslant 1 / 4$ and $G_{0}=\{0\}$. Again, the collection satisfies $2.1(\mathrm{a})-(\mathrm{c})$ but not $2.1(\mathrm{~d})$. Every $f^{-1}\left(G_{t}\right)$ contracts to a point and so

$$
f^{*}: H^{q}\left(G_{t}, A\right) \rightarrow H^{q}\left(f^{-1}\left(G_{t}\right), f^{*} A\right)
$$

is an isomorphism for every $q$ and every $t$, yet $H^{*}(Y, A)$ and $H^{*}\left(X, f^{*} A\right)$ are not isomorphic. Hence, 2.1(a)-(c) are not sufficient for the validity of Corollary 6.4. These sets result from a relation on $Y \times Y$, namely

$$
g(t)= \begin{cases}(0,1] & \text { if } 1 / 4 \leqslant t \leqslant 1 \\ (0, t] & \text { if } 0<t<1 / 4 \\ \{0\} & \text { if } t=0\end{cases}
$$

but this relation is not closed. Hence the conditions of [5] are not satisfied either.

The "strong" converse of the Vietoris-Begle theorem, namely that isomorphisms on the total spaces imply isomorphisms on the sets $G_{t}$, is easily seen to be false. A weak converse theorem will, however, be established. Compare [2, Theorem 4].

Definition 6.6. An $M \subset T$ is a z-set if, for every nontrivial sheaf $\mathscr{A}$ on $T$ concentrated on $M, H^{0}(T, \mathscr{A}) \neq 0$.

EXAMPLE 6.7. Let $M$ be a subset of $T$ such that for every pair $(K, U)$ where $U$ is open in $T$ and $K$ is closed in $U$, and for $t \in K \subset M \cap U$ there is an open set $V$ with

$$
t \in V \subset \bar{V} \subset U \text { and } \partial \bar{V} \cap K=\varnothing .
$$

Then $M$ is a z-set.

Let $\mathscr{A}$ be concentrated on $M$ and let $\mathscr{A}_{t^{\prime}} \neq 0$ for some $t^{\prime} \in M$. Then there is an open set $U$ in $T$ and a section $s$ on $U$ such that $s\left(t^{\prime}\right) \neq 0$. Hence the support $K=|s|$ 
is closed in $U$ and $t^{\prime} \in K \subset M \cap U$. For the open set $V$ satisfying (6.7.1), define $s_{1}$ : $T \rightarrow \mathscr{A}$ by

$$
s_{1}(t)= \begin{cases}s(t) & \text { for } t \in \bar{V}, \\ 0 & \text { for } t \in \tilde{V} .\end{cases}
$$

Since $\bar{V} \cap \tilde{V^{2}}=\partial \bar{V}$ and $\partial \bar{V} \cap K=\varnothing, s_{1}$ is well defined and continuous. Furthermore, $s_{1}\left(t^{\prime}\right)=s\left(t^{\prime}\right) \neq 0$ and so $s_{1}$ is a nonzero member of $\Gamma(T, \mathscr{A})=H^{0}(T, \mathscr{A})$. Consequently, $M$ is a z-set.

This example suggests, when $T$ is paracompact and $M$ has zero relative covering dimension, that $M$ is a z-set. However, in our work, the z-sets enter in $T$ under the topology $\mathfrak{I}_{g}$. The following example exhibits a non-z set, $M$, with $\operatorname{dim}_{T}(M)=0$.

EXAMPLE 6.8. Let $T=\{a, b\}$ with topology $\{\phi,\{a\}, T\}$ and $M=\{a\}$. This topology enters, for example, when $G_{a}$ is a clopen set in $Y$ and $G_{b}=Y$. Let $\mathscr{A}$ be concentrated on $M$. Thus $\mathscr{A}_{a}=H$, an Abelian group, and $\mathscr{A}_{b}=0$. Since the support of any section in $\Gamma(T, \mathscr{A})$ is closed in $T$,

$$
H^{0}(T, \mathscr{A})=\Gamma(T, \mathscr{A})=0 .
$$

Consequently, $M$ is not a z-set. Observe $\Gamma(M, \mathscr{A})=H$.

Let $\mathscr{T}^{0}$ denote the flabby sheaf of serrations into $\mathscr{A}$, with presheaf $\mathscr{T}^{0}(M)=H$ and $\mathscr{T}^{0}(T)=H$ where the restriction homomorphism $r_{T}^{M}: H \rightarrow H$ is the identity. Consequently, we may identify each stalk $\mathscr{T}_{a}^{0}$ and $\mathscr{T}_{b}^{0}$ with $H$, so the sections in $\Gamma\left(T, \mathscr{T}^{0}\right)$ are the constant functions. The inclusion $i: \mathscr{A} \rightarrow \mathscr{T}^{0}$ arises from the presheaf homomorphisms $\Gamma(M, \mathscr{A}) \rightarrow \mathscr{T}^{0}(M)$, the identity homomorphism, and $\Gamma(T, \mathscr{A})=0 \rightarrow \mathscr{T}^{0}(T)$. Hence the sheaf $\mathscr{T}^{1}=\mathscr{T}^{0} / \mathscr{A}$ is concentrated on the closed set $\{b\}$, where $\mathscr{T}_{b}^{1}=H$. Since $\{a\}$ and $T$ are the only nontrivial open sets in $T, \mathscr{T}^{1}$ is clearly flabby. Therefore,

$$
0 \rightarrow \mathscr{A} \rightarrow \mathscr{T}^{0} \stackrel{j}{\rightarrow} \mathscr{T}^{1} \rightarrow 0
$$

is a flabby resolution of $\mathscr{A}$. The cohomology of $\mathscr{A}$ is determined by the chain complex

$$
0 \rightarrow \Gamma\left(T, \mathscr{T}^{0}\right) \stackrel{j^{*}}{\rightarrow} \Gamma\left(T, \mathscr{T}^{1}\right) \rightarrow 0 \rightarrow 0 \rightarrow \cdots,
$$

hence $H^{n}(T, \mathscr{A})=0$ for $n>1$. A section $s \in \Gamma\left(T, \mathscr{T}^{\mathrm{l}}\right)$ is of the form $s(a)=0$, $s(b)=x \in H$ and is the image under $j^{*}$ of the section $s^{\prime}(a)=s^{\prime}(b)=x$. Consequently, $j^{*}$ is surjective whence $H^{1}(T, \mathscr{A})=0$. Since $\mathscr{A}$ represents an arbitrary sheaf concentrated on $M, \operatorname{dim}_{T} M=0$.

The next theorem continues the notation of Theorem 6.3.

THEOREM 6.9. For integers $n<N$ suppose that

$$
\begin{gathered}
d_{q}+q+1 \leqslant n \quad \text { for } 0 \leqslant q \leqslant n-1, \\
M_{q} \text { is a z-set and } d_{q} \leqslant 0 \text { for } n \leqslant q<N,
\end{gathered}
$$

$$
\psi^{*}: H^{q}(Y, \mathscr{A}) \rightarrow H^{q}\left(X, f^{*} \mathscr{A}\right) \text { is an }[n, N] \text { homomorphism. }
$$


Then

$$
\psi_{G_{t}}^{*}: H^{q}\left(G_{t}, \mathscr{A}\right) \rightarrow H^{q}\left(f^{-1}\left(G_{t}\right), f^{*} \mathscr{A}\right)
$$

is an $[n, N)$ homomorphism for all $t$.

Furthermore, if

$$
K_{N}=\left\{t \mid \psi_{G_{t}}^{*}: H^{N}\left(G_{t}, \mathscr{A}\right) \rightarrow H^{N}\left(f^{-1}\left(G_{t}\right), f^{*} \mathscr{A}\right) \text { is not injective }\right\}
$$

is a $\mathrm{z}$-set, then $K_{N}=\varnothing$. Hence in this case $\psi_{G_{t}}^{*}$ is an $[n, N]$ homomorphism for all $t$.

First we verify 5.6(a)-(c), and therefore 5.6(d) and (e), in the present situation. For $p+q=n$ and $p>0, d_{q} \leqslant n-q-1<p$ by (6.9.1). Again (cf. 6.3) the kernel and cokernel of $g(\psi)^{*}$ are concentrated on $M_{q}$. Proposition 6.2 is the authority for the assertion that

$$
\lambda_{2}^{p q}: H^{p}\left(T, \mathscr{H}^{q}(\mathbf{g}, \mathscr{A})\right) \rightarrow H^{p}\left(T, \mathscr{H}^{q}\left(\mathbf{g f}, f^{*} \mathscr{A}\right)\right)
$$

is an epimorphism.

For $n \leqslant q<N, \operatorname{dim}_{T}\left(M_{q}\right) \leqslant 0(6.9 .2)$ and so $\lambda_{2}^{1 q}$ is onto also. Therefore, $\lambda_{2}^{p q}$ is a surjection if $p+q=n$ and $p>0$, or if $n<p+q<N+p$ and $p=1$.

Now consider $n<p+q<N+p, p>1$ and thus $q<N$. For $0 \leqslant q \leqslant n-1$,

$$
d_{q} \leqslant n-q-1<p-1
$$

and for $n \leqslant q<N, d_{q} \leqslant 0<p-1$. Accordingly, $\lambda_{2}^{p q}$ is an isomorphism and implies assertions 5.6(a) and (b), while 5.6(c) is merely the hypothesis (6.9.3).

Define

$$
K_{q}=\left\{t \mid \psi_{G_{t}}^{*}: H^{q}\left(G_{t}, \mathscr{A}\right) \rightarrow H^{q}\left(f^{-1}\left(G_{t}\right), f^{*} \mathscr{A}\right) \text { is not injective }\right\},
$$

a subset of $M_{q}$. Then according to (6.9.2), $K_{q}$ is a z-set for $n \leqslant q<N$ and also for $q=N$ under assumption (6.9.4). If $n<q<N$ and

$$
\mathscr{B}=\operatorname{Ker} \mathbf{g}(\psi)^{*}: \mathscr{H}^{q}(\mathbf{g}, \mathscr{A}) \rightarrow \mathscr{H}^{q}\left(\mathbf{g f}, f^{*} \mathscr{A}\right),
$$

then $\mathscr{B}$ is concentrated on $K_{q}$. The exact sequence

$$
0 \rightarrow \mathscr{B} \stackrel{i}{\rightarrow} \mathscr{H}^{q}(\mathbf{g}, \mathscr{A}) \rightarrow \mathscr{H}^{q}\left(\mathbf{g f}, f^{*} \mathscr{A}\right)
$$

determines an exact sequence

$$
0 \rightarrow H^{0}(T, \mathscr{B}) \stackrel{i^{*}}{\rightarrow} H^{0}\left(T, \mathscr{H}^{q}(\mathbf{g}, \mathscr{A})\right) \stackrel{\lambda_{2}^{0 q}}{\rightarrow} H^{0}\left(T, \mathscr{H}^{q}\left(\mathbf{g}, f^{*} \mathscr{A}\right)\right)
$$

Hence $\operatorname{Im} i^{*}=0$, bearing in mind that $\lambda_{2}^{0 q}$ is one-to-one by $5.6(\mathrm{~d})$. Therefore $H^{0}(T, \mathscr{B})=0$ and, since $\mathscr{B}$ is concentrated on a z-set, $\mathscr{B}=0$. Consequently, $\mathbf{g}(\psi)^{*}$ is a monomorphism and thus, according to $4.7, \psi_{G_{t}}^{*}$ is a monomorphism for all $t$.

Suppose for $n \leqslant q<N$, that

$$
\mathscr{B}=\operatorname{coker} g(\psi)^{*}: \mathscr{H}^{q}(\mathbf{g}, \mathscr{A}) \rightarrow \mathscr{H}^{q}\left(\mathbf{g f}, f^{*} \mathscr{A}\right)
$$


which is concentrated on the $\mathbf{z}$-set $M_{q}$. For $\mathscr{I}=\operatorname{Im}\left(g(\psi)^{*}\right)$, the exact sequence $0 \rightarrow \mathscr{I} \rightarrow \mathscr{H}^{q}\left(\mathbf{g f}, f^{*} \mathscr{A}\right) \rightarrow \mathscr{B} \rightarrow 0$ induces the long exact cohomology sequence

$$
\begin{aligned}
0 & \rightarrow H^{0}(T, \mathscr{I}) \stackrel{\alpha}{\rightarrow} H^{0}\left(T, \mathscr{H}^{q}\left(\mathbf{g f}, f^{*} \mathscr{A}\right)\right) \stackrel{\beta}{\rightarrow} H^{0}(T, \mathscr{B}) \\
& \stackrel{\delta}{\rightarrow} H^{1}(T, \mathscr{I}) \stackrel{\gamma}{\rightarrow} H^{1}\left(T, \mathscr{H}^{q}\left(\mathbf{g f}, f^{*} \mathscr{A}\right)\right) .
\end{aligned}
$$

From the factorization of $g(\psi)^{*}$

$$
\mathscr{H}^{q}(\mathbf{g}, \mathscr{A}) \stackrel{i}{\rightarrow} \mathscr{I} \rightarrow \mathscr{H}^{q}\left(\mathbf{g} \mathbf{f}, f^{*} \mathscr{A}\right)
$$

and the observation that

$$
\lambda_{2}^{0 q}: H^{0}\left(T, \mathscr{H}^{q}(\mathbf{g}, \mathscr{A})\right) \rightarrow H^{0}\left(T, \mathscr{H}^{q}\left(\mathbf{g f}, f^{*} \mathscr{A}\right)\right)
$$

is onto (5.6(d)), follows that $\alpha$ is onto. Accordingly, $\delta$ is a monomorphism.

Next the composition

$$
H^{1}\left(T, \mathscr{H}^{q}(\mathbf{g}, \mathscr{A})\right) \stackrel{i^{*}}{\rightarrow} H^{1}(T, \mathscr{I}) \stackrel{\gamma}{\rightarrow} H^{1}\left(T, \mathscr{H}^{q}\left(\mathbf{g f}, f^{*} \mathscr{A}\right)\right)
$$

is the bijection $\lambda_{2}^{1 q}(5.6(\mathrm{e}))$. Since $i: \mathscr{H}^{q}(\mathrm{~g}, \mathscr{A}) \rightarrow \mathscr{I}$ is an isomorphism except on the set $M_{q}$ of dimension $\leqslant 0, i^{*}$ is onto and therefore $\gamma$ is injective. Hence, $\delta$ is the zero homomorphism, which establishes that $H^{0}(T, \mathscr{B})=0$. Consequently, $\mathscr{B}=0$ and so $\psi_{G_{t}}^{*}$ is an epimorphism for all $t$.

7. The Vietoris-Begle theorem for a relation. The hypotheses of Theorem 6.3 involve hypotheses on the $[0, N)$ singularities which have found expression as dimensional restrictions on the singular sets in $T$. For reasons mentioned earlier such dimensional restrictions may be difficult to verify. We seek therefore to replace $T$ conditions by conditions on subsets of the paracompact space $Y$. This objective is attained under the additional assumption that those singular points contained in the singular $G_{t}$ sets are isolated from the remaining singular points up to dimension $N$. A key technical device is the replacement of an assigned determining collection $\left\{G_{t} \mid t \in T\right\}$ with a more amenable determining collection $\left\{G_{t^{1}}^{1} \mid t^{1} \in T^{1}\right\}$ defined in a canonical way below.

Consider first a special case where a calculable $T$-dimension can be assigned. The result finds immediate application.

Proposition 7.1. Suppose the subset $B \subset T$ consists of a finite number of closed points. Then $B$ is a zero-dimensional $\mathbf{z}-$ set.

First, we show that every sheaf $\mathscr{A}$ on $T$ that is concentrated on $B$ is flabby. Let $s \in \Gamma(U, \mathscr{A})$ and write $B^{1}=B \cap U$. Since $B^{1}$ is closed in $T$ and $s=0$ on $U-B^{1}, s$ extends to $T$.

Hence, for every sheaf $\mathscr{A}$ concentrated on $B, H^{q}(T, \mathscr{A})=0$ for $q>0$ and so $\operatorname{dim}_{T}(B)=0$. Furthermore, if $\mathscr{A} \neq 0$, there is a nonzero section $s \in \Gamma(U, \mathscr{A})$, and by the extension property of $s, H^{0}(T, \mathscr{A}) \neq 0$. Consequently, $B$ is a $\mathbf{z}$-set. 
Proposition 7.2. Assume $Y$ is $[n, N]$ nonsingular and that $\left\{B_{i} \mid i=1,2, \ldots, m\right\}$ is a finite disjoint collection of nonempty closed sets such that every point in the complement of $\bigcup_{i=1}^{m} B_{i}=B$ is $(-1, N)$ nonsingular. Then each $B_{i}$ is $[n, N]$ nonsingular.

Let $S=\{1,2, \ldots, m\}$. Suppose $T$ is the disjoint union of $S$ and $\tilde{B}$, written $T=S+\tilde{B}$. Note that $\tilde{B}$ is open. Let $G_{i}=B_{i}$ for $i \in S$ and $G_{y}=\{y\}$ for $y \in \tilde{B}$. Then $\left\{G_{t} \mid t \in T\right\}$ is a covering of $Y$ by disjoint closed nonempty sets which is a determining collection provided 2.1(c) holds. Consider an open neighborhood $V$ of $G_{t_{0}}$. If $t_{0} \in B^{\tilde{y}}$,

$$
W=\tilde{B} \cap V=\bigcup\left\{G_{t} \mid t \in W\right\}
$$

is an open saturated neighborhood of $G_{t_{0}}$ contained in $W$. If $t=k \in S$, then $B^{1}=\bigcup_{i \neq k} B_{i}$ is closed. Write

$$
W=B^{1^{\sim}} \cap V=\bigcup\left\{G_{t} \mid t \in B^{\tilde{N}} \cap V\right\} \cup G_{k} .
$$

Since the closed set $B_{i}$ has a saturated complement, $g\left(B_{i}\right)=\{i\}$ is closed in $\left(T, \mathfrak{I}_{g}\right)$. Therefore, by 7.1, $S$ is a zero-dimensional z-set. For $y \in \tilde{B}, H^{q}(\{y\}, \mathscr{A})$ $\rightarrow H^{q}\left(f^{-1}(y), f^{*} \mathscr{A}\right)$ is an isomorphism for $0 \leqslant q<N$ by assumption and is a monomorphism for $q=N$. Therefore, $M_{q} \subset S, 0 \leqslant q<N$, and $K_{N} \subset S$ and so $K_{N}$ and each $M_{q}$ is a z-set of dimension $\leqslant 0$. Application of Theorem 6.9 completes the proof of Proposition 7.2.

(7.2.1) The previous proposition underlies the study of $Y=G_{t}$. In particular, if $G_{t}$ is $[n, N]$ nonsingular, $B$ is a closed subset of $G_{t}$ and $U$ is an open neighborhood of $B$ where each point of $U-B$ is $(-1, N)$ nonsingular, then according to $7.2, G_{t}^{1}=G_{t}-$ $U$ and $B$ are $[n, N]$ nonsingular.

Proposition 7.3. Suppose that $\left\{G_{t} \mid t \in T\right\}$ is a determining collection, $B$ is a nonempty closed subset of $Y$, and $U$ is an open neighborhood of $B$. Here and later the set $T_{0}$ enters in a definitive role, where

$$
T_{0}=\left\{t \in T \mid G_{t} \cap \tilde{U^{\sim}} \neq \varnothing\right\} .
$$

Then $\left\{G_{t} \mid t \in T\right\}$ can be replaced by a new determining collection $\left\{G_{t^{1}}^{1} \mid t^{1} \in T^{1}\right\}$, where $T^{1}=T_{0}+(U-B)+\{a\}$, and $a$ is an ideal point, satisfying

$$
\begin{gathered}
G_{a}^{1}=B \\
G_{y}^{1}=\{y\} \quad \text { for } y \in U-B
\end{gathered}
$$

and

$$
G_{t_{0}}^{1}=G_{t_{0}}-U \text { for } t_{0} \in T_{0} .
$$

Furthermore, if the points of $U-B$ are $(-1, N)$ nonsingular, then for $t_{0} \in T_{0}, G_{t_{0}}^{1}$ is $[n, N]$ nonsingular whenever $G_{t_{0}}$ is.

Clearly, $\left\{G_{t^{1}}^{1} \mid t^{1} \in T^{1}\right\}$ is a collection of closed nonempty subsets of $Y$, since $G_{t_{0}}^{1} \neq \varnothing$. For $y \in \tilde{U^{2}}, y \in G_{t}$ for some $t \in T$. Hence, $t \in T_{0}$ and so $y \in G_{t}^{1}$. Consequently, the new collection covers $Y$. 
To verify 2.1(b) it suffices to consider $G_{t_{1}}^{1} \cap G_{t_{2}}^{1}$ for $t_{1}$ and $t_{2}$ in $T_{0}$, since all other intersections are empty. If $y \in G_{t_{1}}^{1} \cap G_{t_{2}}^{1}, y \in U^{\tilde{1}}$ and $y \in G_{t_{1}} \cap G_{t_{2}}$ by (7.3.3). Hence, for some $t_{3} \in T, y \in G_{t_{3}} \subset G_{t_{1}} \cap G_{t_{2}}$. Since $y \in \tilde{U}$, we infer $t_{3} \in T_{0}$ and

$$
y \in G_{t_{3}}^{1}=G_{t_{3}}-U \subset G_{t_{1}}^{1} \cap G_{t_{2}}^{1} .
$$

Therefore, $G_{t_{1}}^{1} \cap G_{t_{2}}$ is $T^{1}$ saturated.

There remains 2.1(c). Let $V$ be an open subset of $Y$. If $G_{a}=B \subset V$, then $B \subset V \cap U \subset V$ where

$$
W=V \cap U=\bigcup\left\{G_{y}^{1} \mid y \in V \cap U-B\right\} \cup B
$$

is an open $T^{1}$ saturated set. For $G_{y_{0}}^{1}=\left\{y_{0}\right\} \subset V$, where $y_{0} \in U-B, W=V \cap(U$ $-B)$ is an open $T^{1}$ saturated neighborhood of $G_{y_{0}}^{1}$ contained in $V$. Finally, for $t \in T_{0}$ and $G_{t}^{1} \subset V$, we infer $G_{t} \subset V \cup U$. (If $V \cap B \neq \varnothing$, replace $V$ with $V-B$.) Accordingly, there is an open $T$-saturated set $W_{1}$ with $G_{t} \subset W_{1} \subset V \cup U$. Hence for $W=W_{1} \cap V, G_{t}^{1} \subset W \subset V$. We now verify that $W$ is $T^{1}$ saturated. If $y \in W$, then $y \notin B$. For $y \in U-B, y \in G_{v}^{1}=\{y\} \subset W$. For $y \in \tilde{U}, t_{1}$ can be found in $T$ for which $y \in G_{t_{1}} \subset W_{1}$. Since $y \in U, t_{1} \in T_{0}$ and therefore

$$
G_{t_{1}}^{1} \subset G_{t_{1}} \subset W_{1} \subset V \cup U .
$$

But $G_{t_{1}}^{1} \cap U=\varnothing$, wherefore, $G_{t_{1}}^{1} \subset V$. Consequently, $y \in G_{t_{1}}^{1} \subset W$. In either case, $W$ is $T^{1}$ saturated.

Therefore, $\left\{G_{t^{1}}^{1} \mid t^{1} \in T^{1}\right\}$ is a determining collection. The last part of Proposition 7.3 is a consequence of (7.2.1).

Proposition 7.4. For $0 \leqslant n<N$, suppose $\left\{G_{t} \mid t \in T\right\}$ is a determining collection, $B$ is a closed subset of $Y$ and $U$ is an open neighborhood of B. Again, take

$$
T_{0}=\left\{t \mid G_{t} \cap \tilde{U^{\sim}} \neq \varnothing\right\} \text {. }
$$

If :

(7.4.1) every point in $U-B$ is $(-1, N)$ nonsingular,

(7.4.2) $G_{t}$ is $(-1, N]$ nonsingular for $t \in T_{0}$, and

(7.4.3) $B$ is $(n-1, N)$ nonsingular,

then $Y$ is $[n, N)$ nonsingular. Furthermore, if $B$ is $(n-1, N]$ nonsingular, then $Y$ is $[n, N]$ nonsingular.

Relying on Proposition 7.3 for justification, replace the collection of $G_{t}$ 's with $\left\{G_{t^{1}}^{1} \mid t^{1} \in T^{1}\right\}$, defined by (7.3.1)-(7.3.3). The set $T_{0}$ is unchanged and the new sets $G_{t_{0}}^{1}$ still satisfy (7.4.2) for $t_{0} \in T_{0}$.

Apply Theorem 6.3 to the new determining collection. For $q<n$ and $t \in M_{q}$,

$$
H^{q}\left(G_{t}^{1}, \mathscr{A}\right) \rightarrow H^{q}\left(f^{-1}\left(G_{t}^{1}\right), f^{*} \mathscr{A}\right)
$$

is not bijective. Hence, $t=a$ by (7.4.1) and (7.4.2) and accordingly $M_{q}$ is either $\{a\}$ or $\varnothing$ for $0 \leqslant q \leqslant n-1$. Since

$$
\tilde{B}=\bigcup_{t_{0} \in T_{0}} G_{t_{0}}^{1} \cup \bigcup_{y \in U-B} G_{y}^{1}
$$


is an open $T^{1}$ saturated set and $G_{a}^{1}=B$ does not intersect any other $G_{t}^{1}$, therefore, $g(B)=\{a\}$ is closed in $\left(T^{1}, \mathfrak{I}_{g^{1}}\right)$. Thus, according to Proposition 7.1, $\operatorname{dim}(\{a\})=0$. Hence, $d_{q} \leqslant 0$ for $0 \leqslant q \leqslant n-1$. Hypotheses (7.4.1)-(7.4.3) ensure that

$$
H^{q}\left(G_{t^{1}}^{1}, \mathscr{A}\right) \rightarrow H^{q}\left(f^{-1}\left(G_{t^{1}}^{1}\right), f^{*} \mathscr{A}\right)
$$

is an isomorphism for $n \leqslant q<N$ and $t^{1} \in T^{1}$, wherefore $\max _{q<N} 1+d_{q}+q \leqslant n$. Accordingly, recourse to Theorem 6.3 establishes our Proposition.

Proposition 7.5. For $0 \leqslant n<N$ and the determining collection $\left\{G_{t} \mid t \in T\right\}$, suppose in the triple $\left(T_{1}, B, U\right)$ that $T_{1} \subset T$ and $B \subset U \subset Y$ where $B$ is closed and $U$ is open and that the following conditions are imposed:

(7.5.1) $G_{t}$ is $[n, N]$ nonsingular for all $t$,

(7.5.2) $G_{t}$ is $(-1, n]$ nonsingular for $t \in T_{1}$,

(7.5.3) $B$ contains all of the $(-1, n)$ singular points of $\bigcup_{t \in T-T_{1}} G_{t}$,

(7.5.4) the points of $U-B$ are $(-1, N)$ nonsingular and

(7.5.5) $B$ is $(n-1, N)$ nonsingular.

Then $Y$ is $[n, N)$ nonsingular. Furthermore, if $B$ is $(n-1, N]$ nonsingular, then $Y$ is $[n, N]$ nonsingular.

First, replace the determining collection with $\left\{G_{t^{1}}^{1} \mid t^{1} \in T^{1}\right\}$, following the precepts of Proposition 7.3. To justify application of Proposition 7.4 to the new determining collection, it is essential to verify (7.4.2). Thus, if $G_{t}$ intersects $\tilde{U}$, then $t \in T_{0}$ and (7.3.3) $G_{t}^{1}=G_{t}-U$.

For $t \in T_{1}, G_{t}$ is $(-1, N]$ nonsingular by (7.5.1) and (7.5.2) and consequently, so is $G_{t}^{1}$ by 7.3. For $t \notin T_{1}, G_{t}^{1}$ contains no $(-1, n)$ singular points by (7.5.3) because $G_{t}^{1} \cap B=\varnothing$. The Vietoris-Begle theorem establishes that $G_{t}^{1}$ is $(-1, n]$ nonsingular. On combining this information with (7.5.1) applied to $G_{t}^{1}$, we can conclude that $G_{t}^{1}$ is $(-1, N]$ nonsingular. In short, condition (7.4.2) holds in either case. Resort to Proposition 7.4 completes the proof of Proposition 7.6.

The culmination of this chain of propositions is our ultimate theorem expressed in terms of dimension of subsets of the paracompact space $Y$, and therefore computable in principle.

THEOREM 7.6. Suppose that each $G_{t}$ is $[n, N]$ nonsingular and that for $T_{1} \subset T, G_{t}$ is $(-1, n]$ nonsingular whenever $t \in T_{1}$. Let $B_{q}$ denote the set of q-singular points in $\bigcup_{t \in T-T_{1}} G_{t}, B=\bigcup_{q=0}^{N-1} B_{q}$ and $d_{q}=r d_{Y} B_{q}$, the relative dimension of $B_{q}$ in $Y$ [8]. If $\bar{B}$ is contained in an open set $U$ where $B$ includes all $(-1, N)$ singular points of $U$ and if

$$
\max _{0 \leqslant q<N} 2+d_{q}+q \leqslant n,
$$

then $\psi^{*}: H^{q}(Y, \mathscr{A}) \rightarrow H^{q}\left(X, f^{*} \mathscr{A}\right)$ is an $[n, N]$ homomorphism.

Since points of $\bar{B}-B$ are $(-1, N)$ nonsingular, $B_{q}$ is the set of $q$-singular points of $\bar{B}$ also. Then, by Theorem 6.3 , with $Y=T=\bar{B}$ and $g=$ the identity function (or by Theorem 1 of [2]), $\bar{B}$ is $(n-1, N]$ nonsingular. Hence, Theorem 7.6 is a direct consequence of 7.5 . 
We can paraphrase Theorem 7.6 as stating that if $\psi_{G_{t}}^{*}$ is an $[n, N]$ homomorphism for all $t$ and a $(-1, N]$ homomorphism for the $t$ 's in $T_{1}$, then $\psi^{*}$ is an $[n, N]$ homomorphism provided that the dimensions of the sets of $q$ singular points contained in the singular sets $G_{t}, t \notin T_{1}$, are sufficiently small for $0 \leqslant q<N$ and furthermore, that these points are isolated from the $(-1, N)$ singular points in the nonsingular sets $G_{t}, t \in T_{1}$. Observe that (7.6.1) asserts $B_{q}=\varnothing$ for $n-1 \leqslant q<N$, i.e. $\bigcup_{t \in T-T_{1}} G_{t}$ does not contain any $q$-singular points in this range.

The theorem covers the situation where an open set $U$ contains both the $(-1, n)$ singular points of $\bigcup_{t \in T-T_{1}} G_{t}$ and the closure of the set of $(-1, N)$ singular points in $U$.

Theorem 7.6 is applicable also if $B$ is the set of $(-1, N)$ singular points in $L=\bigcup_{t \in T-T_{1}} G_{t}$, and if for $U$ the interior of $L, \bar{B} \subset U \subset L$.

\section{BIBLIOGRAPHY}

1. D. G. Bourgin, Cones and Vietoris-Begle type theorems, Trans. Amer. Math. Soc. 174 (1972), 155-183.

2. E. G. Skljarenko, Some applications of the theory of sheaves in general topology, Uspehi Mat. Nauk 19 (1964), 47-49 = Russian Math. Surveys 19 (1964), 41-62. MR30\#1490.

3. D. G. Bourgin, Modern algebraic topology, Macmillan, New York, 1963. MR28\#3415

4. A. P. Wallace, $A$ theorem on acylicity, Bull. Amer. Math. Soc. 67 (1961), 123-124.

5. J. D. Lawson, A generalized version of the Vietoris-Begle theorem, Fund. Math. 65 (1969), 65-72. MR40\#2055

6. Comparison of taut cohomologies, Aequationes Math. 9 (1973), 201-209.

7. G. E. Bredon, Sheaf theory, McGraw-Hill, New York, 1967. MR36\#4552

8. P. Alexandroff, On the dimension of normal spaces, Proc. Roy. Soc. London Ser. A 189 (1947), 11-39.

9. J. Dugundji, Topology, Allyn and Bacon, Boston, Mass., 1966. MR33\#1824

Department of Mathematics, University of Houston, Houston, Texas 77004

Department of Mathematics, TeXas Southern University, Houston, TeXas 77004 